\title{
Broadband spectral analysis of PKS 0528+134: A Report on Six Years of EGRET Observations
}

\author{
R. Mukherjee \\ Dept. of Physics \& Astronomy, Barnard College \& Columbia University, New York, NY 10027 \\ M. Böttcher \\ Space Physics \& Astronomy, Rice University, Houston, TX 77005 \\ R. C. Hartman, P. Sreekumari, \& D. J. Thompson \\ NASA/GSFC, Code 661, Greenbelt, MD 20771 \\ W. A. Mahoney \\ JPL, California Institute of Technology, Pasadena, CA 91109 \\ T. Pursimo, A. Sillanpää, \& L. O. Takalo \\ Tuorla Observatory, University of Turku, SF-21500, Piikkiö, Finland
}

\begin{abstract}
The multiwavelength spectra of PKS $0528+134$ during six years of observations by EGRET have been analyzed using synchrotron self-Compton (SSC) and external radiation Compton models (ERC). We find that a two-component model, in which the target photons are produced externally to the gamma-ray emitting region, but also including an SSC component, is required to suitably reproduce the spectral energy distributions of the source. Our analysis indicates that there is a trend in the observed properties of PKS $0528+134$, as the source goes from a gamma-ray low state to a flaring state. We observe that during the higher gamma-ray states, the bulk Lorentz factor of the jet increases and the ERC component dominates the high-energy emission. Our model calculations indicate the trend that the energies of the electrons giving rise to the synchrotron peak decreases, and the power-ratio of the gamma-ray and low energy spectral components increases, as the source goes from a low to a high gamma-ray state.
\end{abstract}

Subject headings: galaxies: active — galaxies: individual (PKS 0528+134) — gamma rays: observations — radiation mechanisms: nonthermal

\footnotetext{
${ }^{1}$ Universities Space Research Association
} 


\section{Introduction}

PKS 0528+134, a compact radio-loud quasar, is one of the most luminous active galactic nuclei (AGN) detected by the Energetic Gamma Ray Experiment Telescope (EGRET). The source has been observed several times by EGRET, and has also been observed simultaneously at other wavelengths. PKS $0528+134$ is one of the few gamma-ray blazars that has high flux from radio through infrared, and has shown extreme variations in the observed gamma-ray emission. The polarization and flux density of PKS $0528+134$ has been monitored regularly at $4.8 \mathrm{GHz}, 8.0 \mathrm{GHz}$, and 14.5 GHz using the Michigan 26-m telescope (Aller et al. 1985; Aller \& Aller 1996) and has been found to vary on timescales of years. Superluminal motion in PKS 0528+134 was reported previously by Pohl et al. (1995).

Recent findings from geodetic VLBI measurements indicate that outbursts at high frequencies can be linked to structural changes in the radio regime for PKS 0528+134 and some other blazars (Britzen et al. 1998; Krichbaum et al. 1998). VLBI (22-86 GHz) monitoring observations of PKS $0528+134$ by Krichbaum et al. (1998) show pronounced correlated flux density variations in the radio to gamma-ray bands. From their high angular resolution images (0.1-0.2 mas), Krichbaum et al. find increasing evidence for a tight correlation between this activity and the production of new jet components. Similarly, high time-resolution observations of PKS 0528+134 at $8 \mathrm{GHz}$ from the geodetic IRIS campaigns indicate that superluminally moving jet components are ejected preceding the time of an observed gamma-ray flare (Britzen et al. 1998).

PKS 0528+134 is faint in the optical with a mean optical brightness of $m_{v}=19.5$ (Wall $\&$ Peacock 1985). The source is located in the Galactic anticenter $\left(l=191.37^{\circ}, b=-11.01^{\circ}\right)$ behind the diffuse outer edge of the dark cloud B30, in the $\lambda$ Ori ring of clouds (Liszt \& Wilson 1993; Hogerheijde, et al. 1995) and is heavily absorbed. Estimates of Galactic extinction for this

source are very uncertain and range from $A_{v}=2.3$ (Liszt \& Wilson 1993) to $A_{v}=5$, derived from ROSAT observations (Zhang et al. 1994).

There have been few observations of PKS 0528+134 at x-ray energies during the time period of the EGRET observations. Observations with ROSAT were carried out in 1991 (Zhang et al. 1994) and 1992 (Mukherjee et al. 1996). Observations in the medium-hard x-ray range (0.4 - 10 $\mathrm{keV}$ ) were carried out for the first time with ASCA in 1994 and 1995 (Sambruna et al. 1997). RXTE observations of PKS 0528+134 were made during 1996 August and September. Recently, Ghisellini et al. (1998) reported on the BeppoSAX observations of PKS 0528+134 during 1997 February and March.

At gamma-ray energies, PKS 0528+134 has been detected by both OSSE and COMPTEL, besides EGRET. OSSE observed the source to be variable in the $50-150 \mathrm{keV}$ band (McNaronBrown et al. 1995) and COMPTEL observations of the source above $10 \mathrm{MeV}$ were reported by Collmar et al. (1997). The broadband energy spectrum of PKS $0528+134$ is dominated by its gamma-ray emission. Recent analysis of the gamma-ray spectrum of PKS 0528+134 during the 1993 observations indicates the presence of a spectral break between the COMPTEL and EGRET 
energies (Collmar et al. 1997). This can be explained by a variation of the Doppler beaming factor in the framework of a relativistic jet model for blazars (Böttcher \& Collmar 1998).

PKS $0528+134$ is one of the four most variable blazars observed by EGRET (Mukherjee et al. 1997a) and is one of the few blazars seen by EGRET that has flared regularly. Strong variations in flux were observed in 1991, 1993, 1995, and 1996. The highest flux from the source was detected in 1993 March, when the gamma-ray flux was comparable to that of the Crab.

The EGRET results have demonstrated that in order to understand the production of gamma rays in blazars, it is very important to get truly simultaneous coverage across the entire electromagnetic spectrum, before, during, and after a flare in the high-energy gamma-ray emission. The high gamma-ray luminosity of the blazar suggests that the emission is likely to be beamed and, therefore, Doppler-boosted along the line of sight. The jet models explain the radio to UV continuum from blazars as synchrotron radiation from high energy electrons in a relativistically outflowing jet which has been ejected from an accreting supermassive blackhole. The emission in the $\mathrm{MeV}-\mathrm{GeV}$ range is believed to be due to the inverse Compton scattering of low-energy photons by the same relativistic electrons in the jet. However, the source of the soft photons that are inverse Compton scattered remains unresolved. The soft photons can originate as synchrotron emission either from within the jet ("Synchrotron self-Compton" (SSC) mechanism, e. g. Maraschi et al. 1992; Bloom \& Marscher 1996), or from a nearby accretion disk ("External radiation Compton " (ERC) mechanism e. g. Dermer \& Schlickeiser 1994), or they can be disk radiation reprocessed in broad line region (BLR) clouds. The broad-emission-line region in turn can be illuminated either by the disk (Sikora et al. 1994; Blandford \& Levinson 1995), or directly by the jet (Ghisellini \& Madau 1996). An alternative model by Mannheim (1993) proposes that the synchrotron emission is due to the population of both electrons and electron-positron pairs arising from shock-accelerated electrons and protons. Recently, a model combining the ERC and SSC scenarios has been used to fit the simultaneous COMPTEL and EGRET spectra of PKS 0528+134 (Böttcher \& Collmar 1998) measured in Phases 1-3 of the CGRO observations. Böttcher \& Collmar (1998) suggest that in the case of PKS 0528+134, the SSC process dominates the gamma-ray spectrum in the gamma-ray low state, while in the gamma-ray high state an additional ERC component becomes dominant at energies above $10 \mathrm{MeV}$.

In this article we summarize all the information we have on the multiwavelength spectrum of PKS $0528+134$ after six years of observations of the source with EGRET, and examine the SSC and ERC models as applied to PKS $0528+134$. In $\S 4$ we present model calculations to fit the spectrum of PKS 0528+134 during its highest gamma-ray state (1993 March), as well as during its lowest observed gamma-ray state (1996 February - March). We analyze the multiwavelength spectrum of PKS 0528+134 during its "flaring," "intermediate," and "low" states and test if one consistent model fits the spectra during the different states. We start by describing the available multiwavelength data on PKS 0528+134 in $\S 2$. In all the multiwaveband data analyzed here, we have used only simultaneous (or nearly so) data. Although in several cases the samples are not complete, they do provide sufficient information to study the spectral energy distributions of PKS 
$0528+134$ in its various states. The model used for the calculations is described in $\S 3$ and we summarize our results in $\S 5$.

\section{The Data}

\subsection{EGRET Observations}

PKS 0528+134 is one of the 66 blazars detected by EGRET (Hunter et al. 1993; Hartman et al. 1999). Located close to the Crab and Geminga pulsars, there have been several exposures to this source in the past six years of EGRET observations. Table 1 lists the dates of observation and the viewing periods (VPs) during which the source was within $30^{\circ}$ of the EGRET instrument axis. A description of the EGRET instrument, standard data processing techniques, and the maximum likelihood analysis method used to determine the number of source photons are given elsewhere (see for example, Hartman et al. (1999), and the references therein). The integrated fluxes above $100 \mathrm{MeV}$, as well as the significances of detection of PKS $0528+134$ is given in Table 1 for the individual VPs. Some VPs were added together in order to improve the significance of detection. The integrated fluxes from Table 1 are plotted as a function of the observation dates in Figure 1. PKS $0528+134$ is one of the most variable sources seen by EGRET. The source flared to nearly 3 times its mean flux level during the 1993 March observations (VP 213). A $\chi^{2}$ test of the data indicates a probability $<10^{-15}$ that the flux variations are consistent with a constant flux. During the Cycle 6 observations (VP 616.1) PKS 0528+134 was in one of its lowest gamma-ray states ever and was marginally detected at $2.7 \sigma$ during the month-long exposure to the source.

In order to determine the background-subtracted gamma-ray spectrum of PKS 0528+134, the EGRET energy band of $30 \mathrm{MeV}-10 \mathrm{GeV}$ was divided into $10 \mathrm{bins}$, and the number of source photons in each interval was estimated. Details of the spectral analysis technique for EGRET sources may be found in Nolan et al. (1993). The data were fit to a single power law of the form $F(E)=k\left(E / E_{0}\right)^{-\alpha}$ photon $\mathrm{cm}^{-2} \mathrm{~s}^{-1} \mathrm{MeV}^{-1}$, where $F(E)$ is the flux, $E$ is the energy, $\alpha$ is the photon spectral index, $k$ is the coefficient, and $E_{0}$ is the energy normalization factor. Table 2 shows the results of the spectral analysis for PKS 0528+134 in those VPs chosen here for broadband model fits. For the model fits we have chosen a sample of nine VPs with widely varying flux levels. The classification of the gamma-ray state of PKS 0528+134 as "low," "intermediate," or "high" is based on the flux levels and detection significances of the source in the different VPs. Although this scheme of classification is some what arbitrary, it does enable us to compare the spectra of the source between the different observations. In VPs 39, 337, and 616.1, the integrated fluxes above $100 \mathrm{MeV}$ from the source were the lowest in the sample of VPs chosen for the multiwavelength analysis. We designate these observations as "low" states. Similarly, we designate the observations made in VPs $0.2-0.5,213,420$, and 528 as flaring or "high" states. The other VPs analyzed here (VPs 413 and 502) are classified as "intermediate." Although there are other VPs when the source was detected at a high significance, no broadband spectral analysis 
was done because of poor simultaneous multiwavelength coverage. As described further in $\S 3$, it is important to have observations in the optical/IR bands in order to best constrain model fits.

\subsection{Radio Observations}

Historically PKS $0528+134$ has been monitored extensively at radio wavebands. In our analysis we have only included data that are simultaneous, or nearly so, with the EGRET observations. The measurements at $4.8,8.0$, and $14.5 \mathrm{GHz}$ were made with the University of Michigan $26 \mathrm{~m}$ single dish telescope. The data are available on-line from the UMRAO data base (Aller \& Aller 1996). Data at 2.7, 4.8, 10.5 and $32 \mathrm{GHz}$ were obtained from the flux density monitoring program of extragalactic radio sources with the Effelsberg 100-m telescope (Reich et al. 1998). These observations were made using orthogonal cross-scans, and the peak flux densities were derived by fitting Gaussian functions to the observed data. The Effelsberg 100-m telescope was also used to observe the source at 4.85, 10.5 and $23.1 \mathrm{GHz}$ during the EGRET observations in VP 616.1. Data at 22 and $37 \mathrm{GHz}$ were obtained with the $13.7 \mathrm{~m}$ Metsahovi Radio Telescope in Finland (Teräsranta et al. 1992). These observations were made using a dual-beam method and were calibrated against DR21 according to the flux density scale in Baars et al. (1977). The 90 and $230 \mathrm{GHz}$ observations were made with the $15 \mathrm{~m}$ Swedish-ESO Submillimeter Telescope (SEST) on the European Southern Observatory site of La Sille, Chile. These observations are further described in Tornikoski et al. (1996). Observations at 150, 273, and $375 \mathrm{GHz}$ were carried out with the 15-m James Clerk Maxwell Telescope (JCMT) in Hawaii. Radio observations carried out simultaneously with the EGRET measurements in VP 616.1 were reported earlier by Mukherjee et al. (1997b).

\subsection{Optical and Infra-red Observations}

Simultaneous infra-red observations at $J(1.25 \mu \mathrm{m}), H(1.65 \mu \mathrm{m})$, and $K s(2.15 \mu \mathrm{m})$ bands were only available for the EGRET observations in VP 616.1. These observations were carried out during the nights of 1997 February 25 and 26 with the Cassegrain Infrared Camera on the 5-meter telescope at the Palomar Observatory. No night-to-night intensity variations were seen to a limit of a few percent. Optical observations simultaneous with the EGRET observations in VP 213 were made with the $3.5 \mathrm{~m}$ telescope on Calar Alto, Spain (Wagner et al. 1993; Mukherjee et al. 1996). The optical observations of the flare during 1993 March were made two days prior to the EGRET observations. Three one-minute exposures were taken with an $R$-band filter and a Tektronix CCD, and the data were calibrated using a calibration sequence of NGC 2419. Observations at the $B$, $V$, and $R$ bands in 1995 October and 1996 September were made with the $2.5 \mathrm{~m}$ Nordic Optical Telescope (NOT), La Palma, Canary Islands. A BroCamI CCD and TEK $1024 \times 1024$ chip with a pixel size of 0".176 was used for the measurements. Optical data at $R$ and $I$ bands, during the EGRET observations in VP 616.1 were obtained from the figures published in Ghisellini et 
al. (1998). These observations were made at the Torino Astronomical Observatory (TAO), with the $1.05 \mathrm{~m}$ REOSC astrometric telescope equipped with a $1242 \times 1152$ pixel CCD camera. TAO observations at the $R$ band was also made simultaneous with the EGRET observations in VP 502 (Villata et al. 1998). The optical monitoring program of the TAO is further described by Raiteri et al. (1997).

The determination of optical extinction for PKS 0528+134 has always been difficult. Besides the absorption by the column of neutral Galactic hydrogen $\left(N_{H}=(2.6 \pm 0.1) \times 10^{21} \mathrm{~cm}^{-2}\right)$, absorption due to the molecular cloud in the foreground also needs to be taken into account. Here we use a value of $N_{H}=(5.3 \pm 0.1) \times 10^{21} \mathrm{~cm}^{-2}$ for the total column density, estimated by Ghisellini et al. (1998) using archival ASCA observations of the source. This is in good agreement with an earlier estimate of $N_{H} \sim 5 \times 10^{21} \mathrm{~cm}^{-2}$ by Sambruna et al. (1997) using 1994 August ASCA data. Much higher extinctions are suggested by x-ray observations with ROSAT (Zhang et al. 1994). In their earlier analysis of 1993 March data (VP 213), Mukherjee et al. (1996) use a value of $N_{H} \sim 8 \times 10^{21} \mathrm{~cm}^{-2}$, estimated from the ROSAT data. However, this value has large uncertainties, and we have re-analyzed the broadband spectrum of PKS 0528+134 here using the estimate of $N_{H}$ as determined from the ASCA measurements. The extinction was calculated using $A_{\lambda} / E(B-V)=X$ where $X$ is equal to $4.1,3.1,2.32,1.50,0.87,0.54$ and 0.35 for $B, V, R, I$, $J, H$ and $K$ bands, respectively, and $N_{H} / E(B-V)=5 \times 10^{21} \mathrm{~cm}^{-2} \mathrm{mag}^{-1}$ (Savage \& Mathis 1979; Rieke \& Labofsky 1985). The de-reddened fluxes in Jy were estimated using the relation $\operatorname{Flux}(\mathrm{Jy})=A \times 10^{-(0.4 \times \mathrm{mag})}$, where $A$ is the absolute flux density at 0 magnitude, and $\mathrm{mag}$ is the corrected magnitude. The values of $A$ were obtained from the absolute calibration photometry tables in Bessell (1979) (optical) and from Cohen et al. (1992).

\subsection{X-ray Observations}

X-ray observations of PKS 0528+134, and the corresponding EGRET observations (in parentheses) are as follows: ROSAT PSPC observation in 1991 March (VPs 0.2-0.5), and 1992 September (VP 39); ASCA observations in 1994 August (VP 337), and 1995 February and March (VP 413); RXTE observations in 1996 August and September (VP 527 and VP 528); and the BeppoSAX observations in 1997 February and March (VP 616.1). PKS 0528+134 fluxes at $1 \mathrm{keV}$ for the ROSAT observations were obtained from Zhang et al. (1994) and Mukherjee et al. (1996). X-ray fluxes for the ASCA observations were obtained from the published values in Sambruna et al. (1997). We obtained the fluxes for the BeppoSAX observations at the $1 \mathrm{keV}$ band from the historical light curves reported in Ghisellini et al. (1998).

Table 3 shows a list of the observatories contributing to the broad band spectral analysis of PKS 0528+134 presented here. 


\section{The Model}

The model used to fit the spectra of PKS $0528+134$ is described in detail by Böttcher, Mause $\&$ Schlickeiser (1997). Here we only summarize the salient features and outline the assumptions made.

The model for the geometry of the relativistic AGN jet is as follows. The mass of the central black hole is assumed to be $M_{B H}=5 \times 10^{10} M_{\odot}$ and the luminosity of the accretion disk is assumed to be $L=5 \times 10^{46} \mathrm{ergs} \mathrm{s}^{-1}$. In all our calculations we use a Hubble constant $H_{0}=65$ $\mathrm{km} \mathrm{s}^{-1} \mathrm{Mpc}^{-1}, \Lambda=0$, and a $q_{0}=0.5$ cosmology. The model assumes a spherical blob filled with ultrarelativistic pair plasma which is moving with a bulk Lorentz factor $\Gamma$ along an existing jet structure perpendicular to the accretion disk. The angle between the jet axis and the line of sight is $\theta_{\text {obs }}$. The emerging blob starts out at a height of $z_{i}$ (injection height) from the accretion disk. The particles inside the blob at the injection height are distributed isotropically according to the power-law $n(\gamma) \propto \gamma^{-s}$, where $\gamma$ is the Lorentz factor in the rest frame of the blob, ans $s$ is the spectral index. The low and high energy cutoffs in the Lorentz factors of the electrons are given by $\gamma_{1}$ and $\gamma_{2}$. The radio through optical/UV continuum of blazars is explained by synchrotron emission from the plasma in relativistic motion. The electron-positron pair plasma in the blob cools by synchrotron emission and inverse Compton scattering. The model follows the evolution of the pair distribution and the photon spectra as the blob moves out along the jet. The model takes into account synchrotron radiation, SSC and ERC scattering as the various radiative energy loss mechanisms in the AGN jet, and photon-photon absorption via pair production. In the SSC mechanism, the relativistic electrons cool by losing their energy to seed photons originating from the synchrotron emission. In the ERC mechanism, the seed photons are external to the jet, either directly from the accretion disc, or after being re-scattered by the surrounding broad-line region clouds, or both. When making comparisons with the multiwavelength data, the flux of the blob is time-averaged, since the inferred cooling timescale of the plasma in the blob is shorter than the time resolution of the $\gamma$-ray observations.

The model uses a combination of SSC and ERC mechanisms to explain the spectral states of PKS 0528+134 during its various high and low states. A "two-phase" model is supported by the observations that most gamma-ray blazars go through a "quiescent phase" of low flux level and a "flaring phase" when they have a high gamma-ray flux level (Schlickeiser \& Achatz 1992). This has been seen in several cases, e.g. PKS 1622-297 (Mattox et al. 1997), 3C 279 (Wehrle et al. 1998), BL Lac (Bloom et al. 1997). Böttcher \& Collmar (1998) note that a homogeneous SSC model (e.g. Bloom \& Marscher 1996) can explain a smooth transition of the spectrum from hard x-ray and soft gamma-ray energies to a comparatively softer EGRET spectrum. In contrast, in a flaring state, the spectral breaks that are observed between the $\mathrm{MeV}$ energies and the EGRET range can be explained by invoking the ERC mechanism, as such spectral breaks cannot be produced by a pure SSC model. A pure version of either model is therefore probably an unrealistic simplification as it is quite likely that both the SSC and ERC mechanisms are at play during the various states of the AGN. 
The Comptel-EGRET $\gamma$-ray spectra of PKS 0528+134 measured in VPs 1, 213, and 321/322 have been modeled earlier by Böttcher \& Collmar (1998). The spectral variability of the source at $\gamma$-ray energies was explained by these authors by invoking a change in the bulk Lorentz factor $\Gamma$ of the blob. It has been shown by Dermer (1995) and Dermer, Sturner, \& Schlickeiser (1997) that the ERC radiation is related to the Doppler factor as $F_{E R C}(\epsilon) \propto D^{3+s}$, where $D=\left(\Gamma\left[1-\beta_{\Gamma} \cos \theta_{o b s}\right]\right)^{-1}$, while the SSC radiation has a dependence $F_{S S C}(\epsilon) \propto D^{(5+s) / 2}$. Here the bulk Lorentz factor $\Gamma$ is given by $\Gamma=\left(1-\beta_{\Gamma}\right)^{-1 / 2}$. Since the ERC mechanism has a stronger dependence on the Doppler factor than the SSC component, the difference in the spectra between the high and low states was explained by a change in the Doppler factor which translates to a change in $\Gamma$. In the fits presented here, the model has been modified slightly from the version used earlier by Böttcher \& Collmar (1998) so that in addition to $\Gamma$ the particle distribution of the plasma in the blob is also allowed to change between the low and high states. The model parameters that vary are $\Gamma$, the low and high electron energy cutoffs, $\gamma_{1}$ and $\gamma_{2}, n_{e}$, and $s$. The value of the magnetic field $B$ is chosen to be close to the equipartition value. The particles are generally less energetic in the high states, when external inverse Compton scattering becomes more important. This seems plausible since in this case Compton losses are much stronger, impeding the acceleration of electrons to ultrahigh energies. The additional multiwavelength data at optical, infrared, and x-ray energies presented here helps to constrain the model better than the fits to the gamma-ray energies alone, as presented by Böttcher \& Collmar (1998).

Sambruna et al. (1996) have earlier compared the spectral energy distribution (SED) of three complete blazar samples observed by the ROSAT PSPC, namely, RBLs (radio-selected BL Lacs), XBLs (x-ray-selected BL Lacs), and FSRQs (flat-spectrum radio quasars). A clear separation in the radio-through-x-ray SEDs of RBLs and XBLs can be made by defining the objects as LBLs (low-frequency-peaked BL Lacs) or HBLs (high-frequency-peaked BL Lacs) on the basis of their x-ray to radio flux density. Sambruna et al. (1996) have found that the observed differences in the spectral shapes of HBLs, LBLs, and FSRQs correspond to decreasing magnetic field densities, or decreasing electron densities, or both, and cannot be accounted for by a change in the viewing angle solely. In the past we have seen that HBLs can usually be fitted with a pure SSC model with very high electron Lorentz factors (e. g. Mastichiadis \& Kirk (1997) for Mrk 421; Pian et al. (1998) for Mrk 501), while for FSRQs the ERC model generally explains the spectra better (e. g. Böttcher et al. (1997) for 3C 279). Recently Böttcher \& Bloom (1998) have shown that BL Lac, an example of the LBL class, is indeed intermediate between the HBL and FSRQ classes, requiring a relatively weak ERC component. This seems to suggest that generally a higher luminosity is related to a stronger external radiation field, implying stronger inverse Compton losses. It is possible that this trend is also evident in the different states of the same source, as we see in the case of PKS 0528+134 here. 


\section{Multiwavelength Spectral Fits}

In this section we compare the model calculations to the broadband spectrum of PKS $0528+134$. Several observational results help to constrain the choice of parameters in the model fits. VLBI Observations by Pohl et al. (1996) show apparent superluminal motion of at least one component of $\beta_{\text {app }}=4.4 \pm 1.7$ (Pohl et al. 1996), implying that we might reasonably expect $\Gamma>4.4$.

The accretion disk luminosity $L$ is related to the isotropic emission line luminosity, which should be some fraction of $L$. Sambruna et al. (1997) have found $L_{\text {line }}=(0.5-20) \times 10^{46}$ erg $\mathrm{s}^{-1}$, from which we may conclude that $L$ should be greater than a few times $10^{46} \mathrm{erg} \mathrm{s}^{-1}$. The fact that no big blue bump has been observed in PKS $0528+134$ places an upper limit on $L$, which is $\sim 10^{47} \mathrm{erg} \mathrm{s}^{-1}$. An argument for our choice of $M_{B H}\left(>10^{10} M_{\odot}\right)$ here is that the disk luminosity is probably only a rather small fraction $(<10 \%)$ of the Eddington luminosity, because a significant fraction of the accretion power goes into the jets. This implies that $M_{B H} \gg 10^{9} M_{\odot}$. Although the disk luminosity and the black hole mass are not extremely well constrained by the modeling procedure, they cannot be too different from the values adopted here. We estimate that the $L$ and $M_{B H}$ are uncertain by no more than a factor of 2 .

The size of the emission region is constrained by the observed variability of the gamma-ray flux of PKS 0528+134. Short time scale gamma-ray flux variations are detectable by EGRET only in the brightest flaring objects, due to limited photon statistics. In the case of PKS $0528+134$, Mukherjee et al. (1996) report a flux variation by a factor of 2 over a time period $\tau$ of $\sim 2$ days during the 1993 March flare in the source. Doubling times of $\leq 1$ day have been seen in blazars such as 3C 279 (Wehrle et al. 1998) and PKS 1622-297 (Mattox et al. 1997). An upper limit for the extent of the emitting region is given by $R_{B}=c \tau D /(1+z)$ where $D \equiv \Gamma^{-1}\left(1-\beta_{\Gamma} \cos \theta\right)^{-1}$ is the Doppler factor. This gives us an upper limit of $R_{B} \sim 2 \times 10^{15} \mathrm{D} \mathrm{cm}$ for PKS $0528+134$. However, shorter variability timescales with doubling times of $\leq 0.5$ days would imply a higher $\Gamma$ in all states, and the model would have trouble reproducing the observed total luminosity.

Figures 2 through 10 show the multiwaveband spectra of PKS 0528+134 during nine separate EGRET observations of the source. The superimposed solid and broken lines are model calculations, appropriate to reproduce the observed spectra. In each figure the short-dashed line represents the ERC component, the dot-dashed line represents the SSC component, and the solid line is the total flux. The long-dashed line is the synchrotron spectrum and the dotted line represents the accretion disk spectrum. In comparing the model parameters between the nine cases studied here, we note that the quantities describing the physical parameters of the emitting region and the jet are kept the same. The value of $\gamma_{2}$ is nearly the same $\left(\sim 10^{5}\right)$ for all the nine spectra studied here. We discuss the results of the fits to the individual data sets below.

The fits to the spectra in the high or flaring gamma-ray states (VPs 0.2-0.5, 213, 420, and 528) are shown in Figs. 2, 4, 7, and 9, respectively. The fits show that a pure SSC model under-predicts the high energy gamma-ray spectrum. In the case of VP 213, the spectral break 
found by Collmar et al. (1997) cannot be reproduced by the SSC model, and an SSC component with such a high gamma-ray flux would be inconsistent with the x-ray spectrum. A pure ERC model, however, reproduces the high energy gamma-ray spectrum much better than the SSC model, but underpredicts the observed x-ray flux. All the four fits require higher bulk Lorentz factors, which are at least $50 \%$ or more larger than those for the other cases studied here. The values of $\gamma_{1}$ are lower (the stronger cooling implies that the particles are less energetic) than those for the fits to the VPs when the source was not flaring.

The fits to the spectra of PKS 0528+134 in the lowest gamma-ray states (VPs 39, 337, and 616.1) are shown in Figs. 3, 5, and 10, respectively. In two of the three cases the bulk Lorentz factors are the lowest in comparison to the other cases. The fits also require a lower particle density and higher $\gamma_{1}$ compared to the other cases. In the case of VP 337, the Comptel data shown in Fig. 5 were not included in the fit. We were unable to fit the Comptel data with a model that yielded physically meaningful parameters.

The fits to the spectra measured in VPs 413 and 502, when the gamma-ray fluxes and significances of detection are in between the flaring and low states, are shown in Figs. 6 and 8, respectively. In both the VPs the spectral fits require an intermediate value of $\Gamma$, ranging between 7 and 10.

In all the figures the ERC component appears to exceed the total spectrum at high energies. This is because the ERC and SSC components are plotted without taking into account gamma-gamma absorption. The total spectrum is corrected for absorption. This, in fact, shows that gamma-gamma absorption becomes important at high photon energies. The double bump that is observed in the figures in the SSC component is primarily due to second-order SSC scattering. The two-bump structure in the ERC component might be due to the effect of a concave curvature of the evolving electron spectrum, which, in turn, is a consequence of Klein-Nishina effects, leading to less efficient Compton cooling of the highest-energy electrons. This is sometimes also reflected in the curved synchrotron spectrum (e. g., in Figs. 3, 5, and 6).

The fit results are summarized in Table 4. We have not given relative errors on the values of all the parameters, as it was not possible to perform a detailed variational analysis due to the time-consuming nature of our simulations. However, as noted by Böttcher \& Bloom (1998), the self-consistent system simulated here is quite sensitive to changes of the parameters. In some cases some of the parameters like the pair density or $\gamma_{2}$ are not well constrained due to incomplete frequency coverage of the observations. The fit results indicate that a gamma-ray high state is primarily related to an increase of the bulk Lorentz factor $\Gamma$. Hence the ERC component is more dominant during a high gamma-ray state, whereas the SSC component provides the bulk of the high-energy emission during a low gamma-ray state. Also, the particles are generally found to be less energetic in the high states, with lower values of $\gamma_{1}$, which might be related to stronger energy losses by external Compton scattering in this state.

We find that a pure SSC model does not reproduce the spectra well. Strong analytical 
constraints on a pure SSC model fitting the broadband spectrum of PKS 0528+134 can be deduced from the constraints that the intrinsic pair production opacity $\tau_{\gamma \gamma}<1$, and from the total luminosities in the synchrotron and SSC component, assuming that SSC is the only relevant radiation process at $\gamma$-ray energies. The synchrotron and SSC peak frequencies are related by

$$
\epsilon_{S S C} \approx<\gamma^{2}>\epsilon_{s y},
$$

where $\epsilon=h \nu /\left(m_{e} c^{2}\right)$ is the dimensionless photon energy, and the implied isotropic luminosities in both components are

$$
\begin{gathered}
L_{s y} \approx 4 \pi R_{B}^{2} c u_{B}{ }^{\prime}<\gamma^{2}>\tau_{T} D^{4}, \\
L_{S S C} \approx<\gamma^{2}>\tau_{T} L_{s y},
\end{gathered}
$$

where $u_{B}{ }^{\prime}$ is the magnetic field energy density in the blob's comoving frame and $\tau_{T}=n_{e} R_{b} \sigma_{T} \ll 1$ is the Thomson depth of relativistic electrons in the blob. Furthermore, we know that

$$
\epsilon_{s y} \approx \frac{B}{B_{c r}} \frac{D}{1+z}<\gamma^{2}>
$$

where $B_{c r}=4.4 \cdot 10^{13} \mathrm{G}$, and we have the restriction

$$
R_{B} \leq c \Delta t_{\text {var }} \frac{D}{1+z}
$$

from the variability timescale. From Eqs. (1) and (4) we find

$$
u_{B}{ }^{\prime} \approx \frac{B_{c r}^{2}}{8 \pi} \frac{\epsilon_{s y}^{4}}{\epsilon_{S S C}^{2}} \frac{(1+z)^{2}}{D^{2}}
$$

Thus, Eqs. (2) and (3) yield

$$
D^{4} \geq 2 \frac{L_{s y}^{2}}{L_{S S C}} \frac{\epsilon_{S S C}^{2}}{\epsilon_{s y}^{4}} \frac{1}{B_{c r}^{2} c^{3}\left(\Delta t_{v a r}\right)^{2}} .
$$

With peak frequencies $\nu_{s y} \approx 3 \cdot 10^{13} \mathrm{~Hz}$ and $\nu_{S S C} \approx 10^{20} \mathrm{~Hz}$, implied luminosities $L_{s y} \approx 1.3 \cdot 10^{47} \mathrm{erg} \mathrm{s}^{-1}$ and $L_{S S C} \approx 6.3 \cdot 10^{48} \mathrm{erg} \mathrm{s}^{-1}$, and a typical variability time scale of $\sim 2$ days, Eq. (7) gives $D \geq 93$, which appears to be unreasonably high. Apart from a very high Lorentz factor $\Gamma \geq 50$, it would require extremely close alignment of the jet with respect to the line of sight: $\theta_{\text {obs }} \leq 0.6^{\circ}$. It would also require the magnetic field to be very weak, $B<0.13 \mathrm{G}$ (Eq. [4]), implying a very strong deviation from equipartition:

$$
\frac{u_{B}}{u_{e}} \leq \frac{B_{c r}^{2}}{8 \pi m_{e} c^{2}} \frac{\epsilon_{s y}^{7 / 2}}{\epsilon_{S S C}^{3 / 2}} \frac{L_{s y}}{L_{S S C}} \sigma_{T} c \Delta t_{v a r} \approx 0.03 .
$$


The estimate (7) is much more severe than the constraint resulting from the estimate of the $\gamma \gamma$ opacity, which also applies to the external inverse-Compton mechanism:

$$
1<\tau_{\gamma \gamma} \approx \frac{L_{\gamma} \sigma_{T}(1+z)}{4 \pi m_{e} c^{4} \Delta t_{\text {var }} D^{5}},
$$

which yields $D>6$ for the values of the observables as quoted above.

A fit of a pure SSC model to VP 0.2-0.5 (Fig. 11), using parameters as deduced in the above analytical estimates shows that (in addition to the rather implausibly high $\Gamma$ and small angle $\left.\theta_{\text {obs }}\right)$ this model has problems with the low x-ray flux, and the IR flux seems too high. However, the gamma-ray spectrum is quite well reproduced by this calculation. The model parameters used for this calculation are $\gamma_{1}=2 \times 10^{3}, \gamma_{2}=5 \times 10^{4}, s=2.5, n_{e}=200 \mathrm{~cm}^{-3}, B=0.13 \mathrm{G}$, $\Gamma=95$, and $\theta_{o b s}=0.6^{\circ}$. An injection height $z_{i}=10 \mathrm{pc} \gg R_{B}$ was needed in order to suppress the ERC component, and is not a very realistic number either. Figure 12 shows a pure SSC model calculation to the data in VP 616.1. In this case, however, the fit is not much worse than the SSC/ERC model calculation shown in Fig. 10. The parameters obtained are $\gamma_{1}=3 \times 10^{3}$, $\gamma_{2}=2 \times 10^{4}, s=2.8, n_{e}=100 \mathrm{~cm}^{-3}, B=3.3 \mathrm{G}, \Gamma=10$, and $z_{i}=1 \mathrm{pc}$.

Sambruna et al. (1997) observe that the relative contributions of the SSC and ERC cooling mechanisms depends on the gamma-ray to optical flux ratio for a source. They have analyzed the low state data of PKS 0528+134 during the 1994 August observations (VP 337), and note that even in the low state the gamma-ray flux exceeds the optical flux of the source, indicating that the ERC scattering may be the primary cooling mechanism. Our findings are similar to the results of Sambruna et al. (1997). In all the spectra that we have analyzed which have optical observations, the gamma-ray flux exceeds the optical flux from the source. This is true even for VP 616.1, when the source was in its historically lowest gamma-ray state. Hence it is not surprising that there is a significant contribution from the ERC scattering to the model fit of the VP 616.1 spectrum. Because of its lower gamma-ray to optical flux ratio than for the other VPs studied, the SSC mechanism also plays a major role in the cooling of the relativistic electrons during VP 616.1. In comparison, in VP 213, when the gamma-ray to optical flux ratio was the highest ever, a pure SSC model yields a very poor fit to the data, and the contribution of the ERC mechanism is significantly larger.

Recently, Ghisellini et al. (1998) have studied the spectral energy distributions of 51 EGRET-detected blazars, and have applied the SSC and ERC models to the spectra of these sources. In their analysis the ERC model includes contributions from the SSC component, and can therefore be compared with our analysis presented here. Although their broadband spectra did not include simultaneous measurements at all frequencies, they were able to determine trends and correlations among the physical quantities obtained from their model calculations. Ghisellini et al. (1998) find evidence for a well-defined sequence in the properties of different blazar classes, namely, HBL, LBL, HPQ (high-polarization quasars), and LPQ (low-polarization quasars). They find that the observed spectral properties of these source classes can be explained by an increasing 
contribution of an external radiation field as we look at the sequence HBL $\rightarrow$ LBL $\rightarrow$ HPQ $\rightarrow$ LPQ. The trends in the observed properties are a decrease in frequencies of the synchrotron and inverse Compton peaks, and an increase in the power-ratio of the high and low energy spectral components. In our analysis here, we see a similar trend in the physical properties of the different spectral states of PKS $0528+134$. As we study the sequence of flaring, intermediate, and low states

of the source, we see that the model accounts for the flaring state better with a lower $\gamma_{1}$ (decrease in the synchrotron peak) and a higher ratio of synchrotron to gamma-ray luminosity. The bulk Lorentz factor for the flaring states is found to be higher than that in the non-flaring states, in agreement with the trend found by Ghisellini et al. (1998). The Lorentz factor is an important discriminant in the comparisons between the different spectral states, because it determines the location of the synchrotron and inverse Compton peaks. Although the synchrotron peak for PKS $0528+134$ is not well-constrained by the data presented here, our conclusions about $\gamma_{1}$ result primarily from the x-ray flux (which is SSC emission from low-energy electrons with energies around $\gamma_{1}$ ) and from the spectral break at $\mathrm{MeV}$ energies.

The model used here explains the broadband spectrum of PKS 0528+134 from optical to high energy gamma rays reasonably well, but underpredicts the radio flux. This is because the low energy cutoff in the electron energy produces a sharp cutoff in the synchrotron spectrum below $\sim 10^{13} \mathrm{~Hz}$ where the blob becomes optically thick to synchrotron-self absorption. The radio flux only varies with very small amplitude. Thus it is most probably produced in the outer jet regions which are less influenced by single blob injection events. The outer jet regions, where the kinetic power of the jet is dissipated and the expansion of the jet becomes relevant, are not included in our simulations.

The Böttcher \& Collmar (1998) model generally has problems with the hard optical spectra which resemble more the instantaneous rather than the time-averaged synchrotron spectra of the evolving electron population. However, recently Chiaberge \& Ghisellini (1998) have noted that even if the electron cooling time scale is much shorter than the light travel time through the blob, no strong variability results on a time scale shorter than the light travel time. Thus it is likely that we are seeing the time-averaged spectrum even in the optical. Alternatively, it is possible that reacceleration at the low-energy end of the electron spectrum during the early stages of the blob evolution would lead to an optical spectrum resembling the instantaneous spectrum of the source.

\section{Summary \& Conclusions}

We present model calculations of the multiwavelength spectrum of PKS 0528+134 during its various gamma-ray states. The broad-band spectrum of PKS $0528+134$ is dominated by the emission in gamma rays. This is true even when the source was in its lowest ever gamma-ray state.

We find that the spectral energy distribution of PKS $0528+134$ can be modeled as follows: the radio to UV emission can be explained as synchrotron emission from relativistic electrons 
in a uniform relativistically moving plasma. The high energy emission is due to the inverse Compton scattering of seed photons off the relativistic electrons. The electron cooling process is a combination of SSC and ERC mechanisms, based on the different dependences of the ERC and the SSC components on the Doppler boosting factor. In the gamma-ray high state, due to an increase in the bulk Lorentz factor of the jet, the ERC component becomes the only significant part of the gamma-ray spectrum.

The model that yields an acceptable fit to the data assumes the mass of the central black hole $M_{B H}=5 \times 10^{10} M_{\odot}$ and the luminosity of the accretion disk to be $L=5 \times 10^{46} \mathrm{ergs} \mathrm{s}^{-1}$ for PKS $0528+134$.

It has generally been seen that sources with higher bolometric luminosity, dominated more strongly by the gamma-ray luminosity, are fitted better with the ERC model rather than a pure SSC model. Ghisellini et al. (1998) find that the properties of HBL, LBL, and FSRQs are located along a sequence, with the HBL characterized by the lowest intrinsic power and weakest external radiation field. Our model calculations indicate that a similar trend exists in the flaring and quiescent spectral states of PKS 0528+134. It would be interesting to test if this is generally true by analyzing the flaring and quiescent spectra of other blazars detected by EGRET that have exhibited similar extreme variations in their gamma-ray flux (e. g. 3C 279, PKS 1622-297).

The spectral variability in PKS 0528+134 appears to arise from the different Doppler boosting patterns of the SSC and the ERC radiations. The relative contributions of the SSC and ERC cooling mechanisms seem to be related to the optical to gamma-ray flux ratio from the source. The SSC mechanism plays a larger role if the source is in a low flux state. The ERC mechanism is the dominant cooling mechanism when the source is in a high gamma-ray state.

The main source of uncertainty in the model parameters arises from incomplete spectral coverage of PKS 0528+134 during the various observation periods. More precise (contemporaneous) measurements of the high-energy cutoffs of the synchrotron (in the UV) and gamma-ray spectral components are necessary to constrain models better, especially parameters like the high-energy cutoff of the electron spectrum. Future observations of AGN with successor gamma-ray observatories like INTEGRAL (e.g. Lichti et al. 1996) and GLAST (e.g. Michelson 1996) should play a key role in resolving the physics of these powerful sources.

R. Mukherjee acknowledges support by NASA Grant NAG5-3696. This research has made use of data from the University of Michigan Radio Astronomy Observatory which is supported by the National Science Foundation and by funds from the University of Michigan. M. Böttcher acknowledges support by NASA Grant NAG5-4055. R. Mukherjee acknowledges many useful discussions with J. Halpern. 


\section{REFERENCES}

Aller, H. D., et al. 1985, ApJ, 59, 513.

Aller, H. D. \& Aller M. 1996, AAS 189th meeting. Also see URL: http://www.astro.lsa.umich.edu/.

Baars, J. W. M., et al. 1977, A\&A, 61, 99.

Bessell, M. S. 1979, P. A. S. P., 91, 589.

Blandford, R. D. \& Levison, A. 1995, ApJ, 441, 79

Bloom, S. D. \& Marscher, A. P. 1996, ApJ, 461, 657

Bloom, S. D., et al. 1997, ApJ, 490, L145.

Böttcher, M., et al. 1997, CP410, Proceedings of the Fourth Compton Symposium edited by C. D. Dermer, M. S. Strickman, and J. D. Kurfess, pg. 1473.

Böttcher, M., Mause, H., \& Schlickeiser, R. 1997, A\&A, 324, 395.

Böttcher, M. \& Collmar, W. 1998, A\&A, 329, L57.

Böttcher, M. \& Bloom, S. D. 1998, ApJ, submitted.

Britzen, S. et al. 1998, Radio Emission from Galactic and Extragalactic Compact Sources, ASP Conference Series, Volume 144, IAU Colloquium 164, eds. J.A. Zensus, G.B. Taylor, \& J.M. Wrobel, p. 43.

Chiaberge, M. \& Ghisellini, G. 1998, MNRAS, submitted; astro-ph/9810263.

Cohen, M., et al. 1992, AJ, 104, 1650.

Collmar, W., et al. 1997, A\&A, 328, 33.

Dermer, C. D. \& Schlickeiser, R. 1994, ApJS, 90, 945

Dermer, C. D. 1995, ApJ, 446L, 63.

Dermer, C. D., Sturner, S. J., \& Schlickeiser, R. 1997, ApJS, 109, 103.

Ghisellini, G. \& Madau, P. 1996, MNRAS, 280, 67

Ghisellini, G., et al. 1998, Proc. of SAX workshop.

Ghisellini, G., et al. 1998, submitted to M. N. R. A. S.

Hartman, R. C., et al. 1997, CP410, Proceedings of the Fourth Compton Symposium edited by C. D. Dermer, M. S. Strickman, and J. D. Kurfess, pg. 307.

Hartman, R. C., et al. 1999, ApJ, in press.

Hogerheijde, M., et al. 1995, ApJ, 441, L93.

Hunter, S. D., et al. 1993, ApJ, 409, 134.

Krichbaum, T. P., et al. 1998, Radio Emission from Galactic and Extragalactic Compact Sources, ASP Conference Series, Volume 144, IAU Colloquium 164, eds. J.A. Zensus, G.B. Taylor, \& J.M. Wrobel, pg. 37. 
Lichti, G. G., et al. 1996, SPIE, 2806, 217.

Liszt, H. S., \& Wilson, R. W. 1993, ApJ, 403, 663.

Mannheim, K., 1993, A\&A, 269, 67.

Maraschi, L., Ghisellini, G., \& Celotti, A. 1992, ApJ, 397, L5

Mastichiadis, A. \& Kirk, J. G. 1997, A\&A, 320, 19.

Mattox, J. R., et al. 1997, ApJ, 476, 692.

McNaron-Brown, K., et al. 1995, ApJ, 451, 575.

Michelson, P. F. 1996, SPIE, 2806, 31.

Mukherjee, R., et al. 1997a, ApJ, 490, 116.

Mukherjee, R., et al. 1997b, CP410, Proceedings of the Fourth Compton Symposium edited by C. D. Dermer, M. S. Strickman, and J. D. Kurfess, pg. 1346.

Mukherjee, R., et al. 1996, ApJ, 470, 831.

Nolan, P. et al. 1993, ApJ, 409, 697.

Pian, E., et al. 1998, ApJ, 492L, 17.

Pohl, M., et al. 1995, A\&A, 303, 383.

Pohl, M., et al. 1996, A\&AS, 120, 529.

Raiteri, C. M., et al. 1997, A\&A Supplement Series, 127, 445.

Reich, W., et al. 1998, A\&AS, 131, 11.

Rieke, G. H. \& Labofsky, J. H. 1985, ApJ, 288, 618.

Sambruna, R., et al. 1997, ApJ, 474, 639.

Savage, B. D. \& Mathis, J. S., 1979, ARAA, 17, 73.

Sikora, M., Begelman, M. C., \& Rees, M. J. 1994, ApJ, 421, 153

Schlickeiser, R., \& Achatz, U. 1992, in: Extragalactic Radio Sources - From Beams to Jets, eds. J. Roland, H. Sol, G. Pelletier, Cambridge University Press, Cambridge, p. 214.

Teräsranta, H., et al. 1992, A\&AS, 94, 121.

Tornikoski, M., et al. 1996, A\&AS, 116, 157.

Villata, M., et al. 1998, A\&A Supplement Series, 121, 119.

Wagner, S., et al. 1993 (priv. comm.).

Wall, J. V., \& Peacock, J. A. 1985, MNRAS, 216, 173.

Wehrle, A. E., et al. 1998, ApJ, 497, 178.

Zhang, Y. F., et al. 1994, ApJ, 432, 91. 
Table 1. EGRET observations of PKS 0528+134 from 1991 to 1997

\begin{tabular}{|c|c|c|c|c|}
\hline $\begin{array}{l}\text { Viewing } \\
\text { Period }\end{array}$ & $\begin{array}{c}\text { Observation } \\
\text { Dates }\end{array}$ & $\begin{array}{l}\text { Flux } \times 10^{-7} \\
\text { ph } \mathrm{cm}^{-2} \mathrm{~s}^{-1}\end{array}$ & $\begin{array}{l}\text { Significance } \\
\qquad \sigma\end{array}$ & $\begin{array}{c}\text { Inclination Angle } \\
\text { of Source }\end{array}$ \\
\hline $0.2-0.5$ & 1991 Apr 22 - May 07 & $12.9 \pm 0.9$ & 20.3 & $8 .^{\circ} 0$ \\
\hline 1.0 & 1991 May $16-30$ & $8.5 \pm 0.8$ & 13.5 & $6 .{ }^{\circ} 3$ \\
\hline 2.1 & 1991 Jun $08-15$ & $3.6 \pm 1.1$ & 3.8 & $5 .^{\circ} 1$ \\
\hline $36.0+36.5$ & 1992 Aug $11-20$ & $<5.5^{a}$ & - & $22 .^{\circ} 0$ \\
\hline 39.0 & 1992 Sep $01-17$ & $3.2 \pm 1.4$ & 2.6 & $23 .^{\circ} 9$ \\
\hline 213.0 & 1993 Mar $23-29$ & $30.8 \pm 3.5$ & 13.6 & $9 .^{\circ} 1$ \\
\hline 221.0 & 1993 May $13-24$ & $2.3 \pm 1.2$ & 2.2 & $6 .{ }^{\circ} 4$ \\
\hline 310.0 & 1993 Dec $01-13$ & $<4.0^{\mathrm{a}}$ & - & $15 .^{\circ} 7$ \\
\hline $321.1+321.5$ & 1994 Feb $08-17$ & $4.9 \pm 1.2$ & 5.0 & $12 .^{\circ} 9$ \\
\hline 337.0 & 1994 Aug $09-29$ & $3.2 \pm 1.0$ & 3.6 & $13 .^{\circ} 5$ \\
\hline 412.0 & 1995 Feb 28 - Mar 07 & $9.1 \pm 2.1$ & 5.8 & $13 .^{\circ} 1$ \\
\hline 413.0 & 1995 Mar $07-21$ & $9.0 \pm 1.3$ & 9.3 & $7 .^{\circ} 7$ \\
\hline 419.1 & 1995 Apr $04-11$ & $12.1 \pm 2.4$ & 6.6 & $17 .^{\circ} 4$ \\
\hline 419.5 & 1995 May $09-23$ & $12.0 \pm 2.2$ & 7.5 & $20 .^{\circ} 9$ \\
\hline 420.0 & 1995 May 23 - Jun 06 & $13.0 \pm 1.6$ & 11.2 & $9 .^{\circ} 8$ \\
\hline 426.0 & 1995 Aug $08-22$ & $5.5 \pm 1.7$ & 4.0 & $8 .^{\circ} 5$ \\
\hline 502.0 & 1995 Oct $17-31$ & $5.7 \pm 0.8$ & 8.5 & $0 .{ }^{\circ} 9$ \\
\hline 526.0 & 1996 Jul 30 - Aug 13 & $5.2 \pm 1.2$ & 5.4 & $8 .{ }^{\circ} 3$ \\
\hline 527.0 & 1996 Aug $13-20$ & $5.1 \pm 1.6$ & 4.3 & $9 .{ }^{\circ} 3$ \\
\hline 528.0 & 1996 Aug $20-27$ & $17.3 \pm 2.1$ & 11.7 & $12 .^{\circ} 1$ \\
\hline 616.1 & 1997 Feb 18 - Mar 18 & $1.1 \pm 0.5$ & 2.7 & $0 .{ }^{\circ} 0$ \\
\hline
\end{tabular}

a: $2 \sigma$ upper limit 


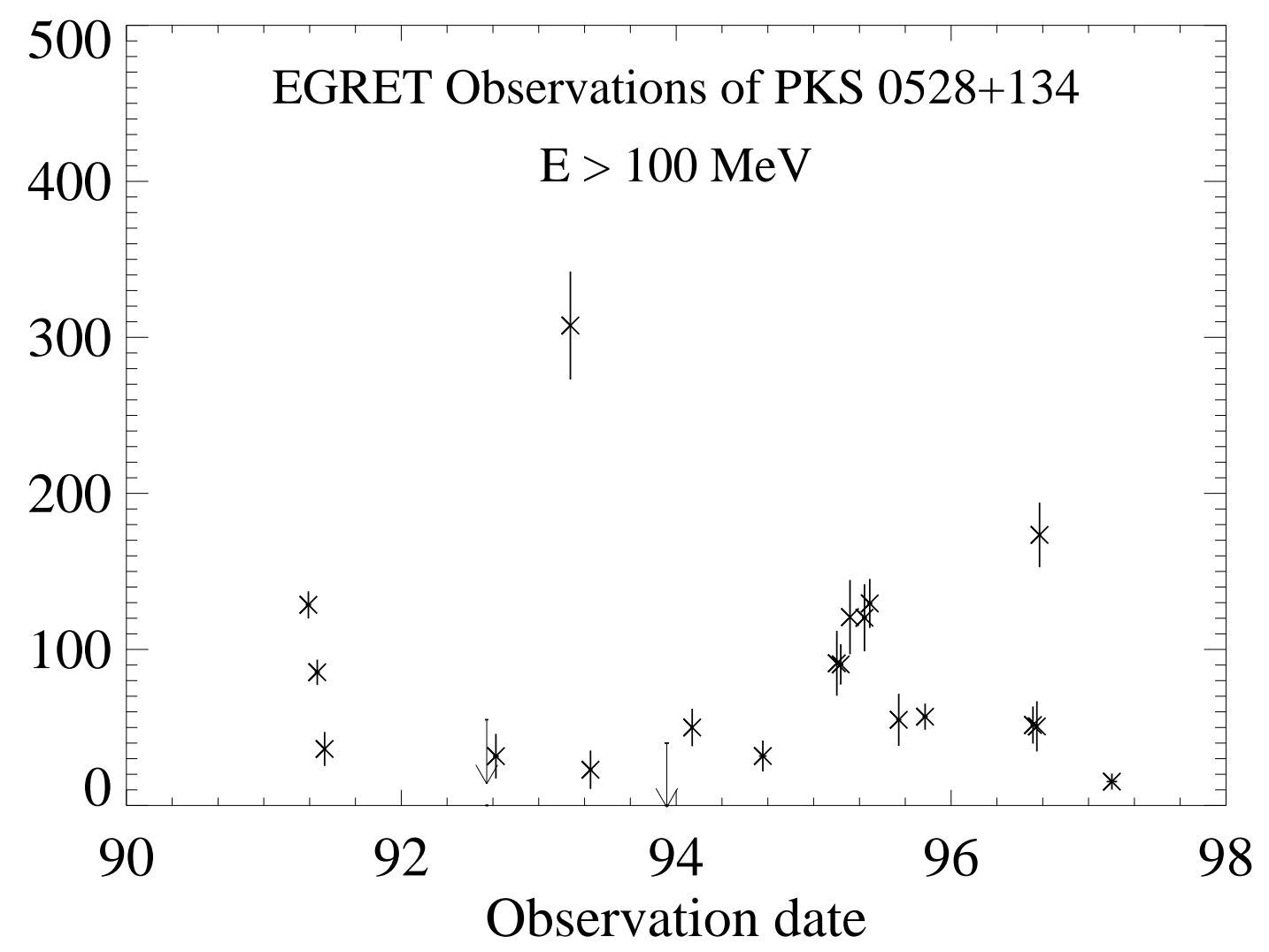

Fig. 1.- Flux of gamma rays above $100 \mathrm{MeV}$ from PKS 0528+134 over the period 1991 April to 1997 March (Phases 1 through Cycle 6). 2 $\sigma$ upper limits are shown as downward arrows. 
Table 2. EGRET spectral analysis for PKS 0528+134 in selected observations

\begin{tabular}{lcccc}
\hline \hline $\begin{array}{l}\text { Viewing } \\
\text { Period }\end{array}$ & $\begin{array}{c}\text { Spectral } \\
\text { Index }(\alpha)\end{array}$ & $\begin{array}{c}\mathrm{k} \times 10^{-9} \\
\mathrm{ph} \mathrm{cm}^{-2} \mathrm{~s}^{-1} \mathrm{MeV}^{-1}\end{array}$ & $\begin{array}{c}E_{0} \\
\mathrm{MeV}\end{array}$ & $\chi^{2} / n_{f}$ \\
& & & & \\
\hline $0.2-0.5$ & $2.27 \pm 0.07$ & $3.26 \pm 0.19$ & 199 & 1.53 \\
39.0 & $2.39 \pm 0.78$ & $1.17 \pm 0.61$ & 166 & 0.13 \\
213.0 & $2.21 \pm 0.11$ & $8.24 \pm 0.76$ & 200 & 0.78 \\
337.0 & $2.68 \pm 0.44$ & $2.15 \pm 0.57$ & 135 & 0.52 \\
413.0 & $2.21 \pm 0.16$ & $0.92 \pm 0.15$ & 255 & 1.07 \\
420.0 & $2.37 \pm 0.13$ & $2.48 \pm 0.29$ & 206 & 1.19 \\
502.0 & $2.32 \pm 0.16$ & $1.16 \pm 0.17$ & 235 & 0.55 \\
528.0 & $2.44 \pm 0.44$ & $2.73 \pm 0.76$ & 159 & 0.19 \\
616.1 & $2.51 \pm 0.47$ & $0.22 \pm 0.10$ & 233 & 0.76 \\
\hline
\end{tabular}


Table 3. Contributing observatories and spectral coverage

\begin{tabular}{llll}
\hline \hline Observatory & Instrument/Telescope & Spectral region & \multicolumn{1}{c}{ band } \\
& & & \\
\hline \multirow{2}{*}{ CGRO } & EGRET & $\gamma$-ray & $0.03-10 \mathrm{GeV}$ \\
& COMPTEL & $\gamma$-ray & $0.75-30 \mathrm{MeV}$ \\
ROSAT & PSPC & x-ray & $0.5-2 \mathrm{keV}$ \\
ASCA & & x-ray & $0.5-2 \mathrm{keV}$ \\
Beppo SAX & & x-ray & $0.5-2 \mathrm{keV}$ \\
Palomar & $5 \mathrm{~m}$ & infra red & $J H \mathrm{H}$ \\
Torino & $1.05 \mathrm{~m}$ & optical & $R I$ \\
NOT & $2.56 \mathrm{~m}$ & optical & $B V R$ \\
Calar Alto & $3.5 \mathrm{~m}$ & optical & $R$ \\
Effelsberg & $100 \mathrm{~m}$ & mm/cm & $2.69,4.8,10.5,23.1,32 \mathrm{GHz}$ \\
SEST & $15 \mathrm{~m}$ & mm & $90,230 \mathrm{GHz}$ \\
Metsähovi & $13.7 \mathrm{~m}$ & mm/cm & $22,36.8 \mathrm{GHz}$ \\
JCMT & $15 \mathrm{~m}$ & mm/sub-mm & $150,273,375 \mathrm{GHz}$ \\
UMRAO & $26 \mathrm{~m}$ & $\mathrm{~cm}$ & $4.8,8.0,14.5 \mathrm{GHz}$ \\
\hline
\end{tabular}


Table 4. Interesting model parameters for the different viewing periods

\begin{tabular}{cccccccl}
\hline \hline VP & $\gamma_{1}$ & $\gamma_{2}$ & $s$ & $\Gamma$ & $\begin{array}{c}n_{e} \\
\mathrm{~cm}^{-3}\end{array}$ & $\begin{array}{c}B \\
\mathrm{G}\end{array}$ & $\begin{array}{l}\text { Gamma-ray } \\
\text { State* }\end{array}$ \\
& & & & & & & \\
\hline $0.2-0.5$ & 180 & $10^{5}$ & 2.5 & 15 & 290 & 2.5 & High \\
39.0 & 1000 & $10^{5}$ & 2.5 & 5 & 150 & 2.5 & Low \\
213.0 & 400 & $6 \times 10^{4}$ & 2.5 & 20 & 180 & 2.0 & High \\
337.0 & 800 & $10^{5}$ & 2.5 & 10 & 150 & 3.0 & Low \\
413.0 & 1000 & $10^{5}$ & 2.5 & 10 & 180 & 2.0 & Intermediate \\
420.0 & 500 & $7 \times 10^{4}$ & 2.5 & 20 & 180 & 2.0 & High \\
502.0 & 1000 & $10^{5}$ & 2.5 & 7 & 180 & 3.2 & Intermediate \\
528.0 & 600 & $10^{5}$ & 2.2 & 10 & 180 & 2.5 & High \\
616.1 & 1000 & $10^{5}$ & 2.5 & 5 & 50 & 2.5 & Low \\
& & & & & & & \\
\hline
\end{tabular}

* The high energy gamma-ray state of the source (EGRET energy range), based on the criteria described in the text. 


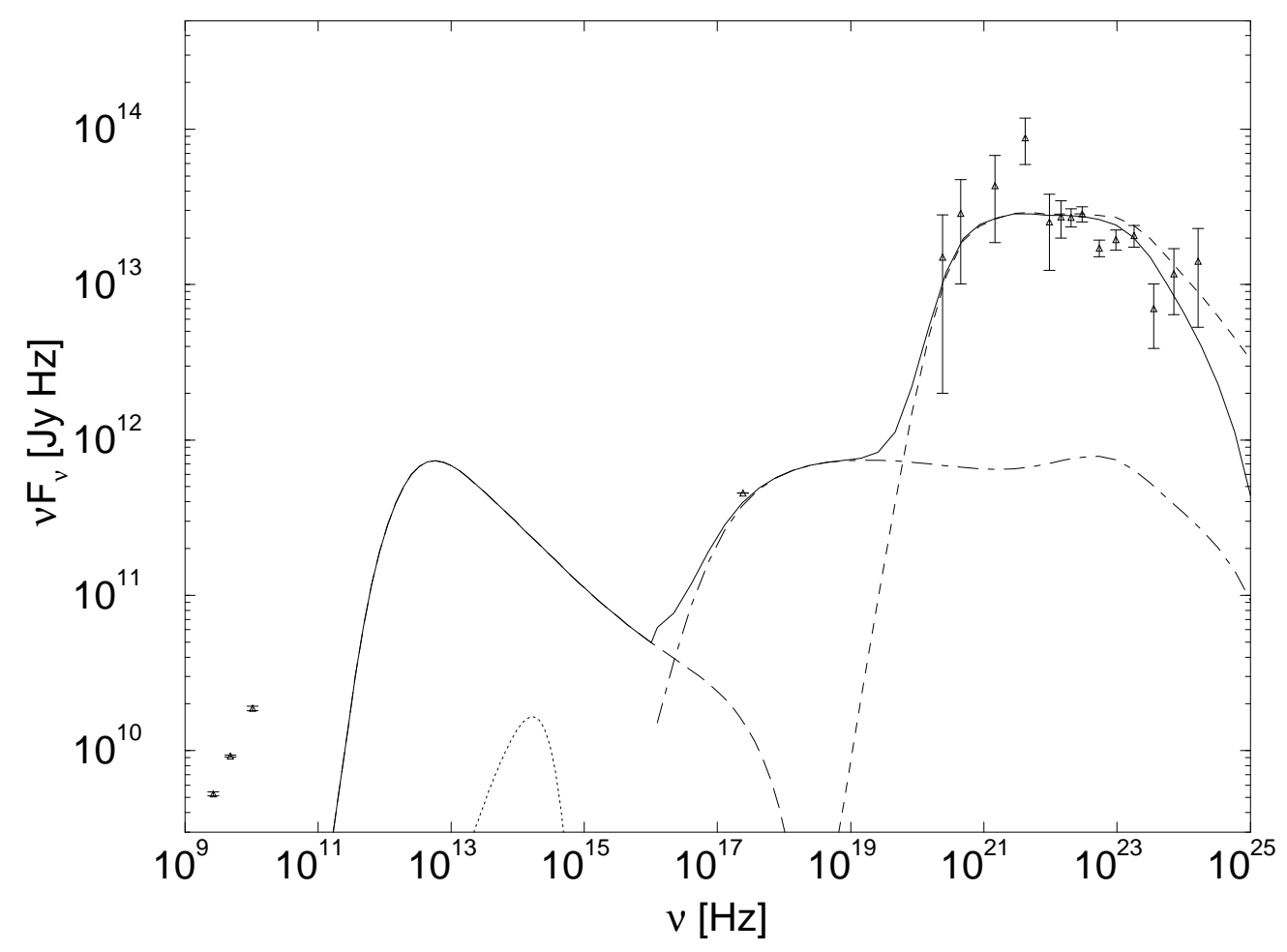

Fig. 2. - Fit to the spectral energy distribution of PKS 0528+134 in VP 0.2-0.5. The long-dashed line represents the synchrotron spectrum, the short-dashed line is the ERC component, the dotdashed line is the SSC component, and the solid line represents the combined SSC + ERC model. The dotted line is the accretion disk spectrum. Parameters: $\gamma_{1}=180, \gamma_{2}=10^{5}, s=2.5, n_{e}=290$ $\mathrm{cm}^{-3}, B=2.5 \mathrm{G}, \Gamma=15$. 


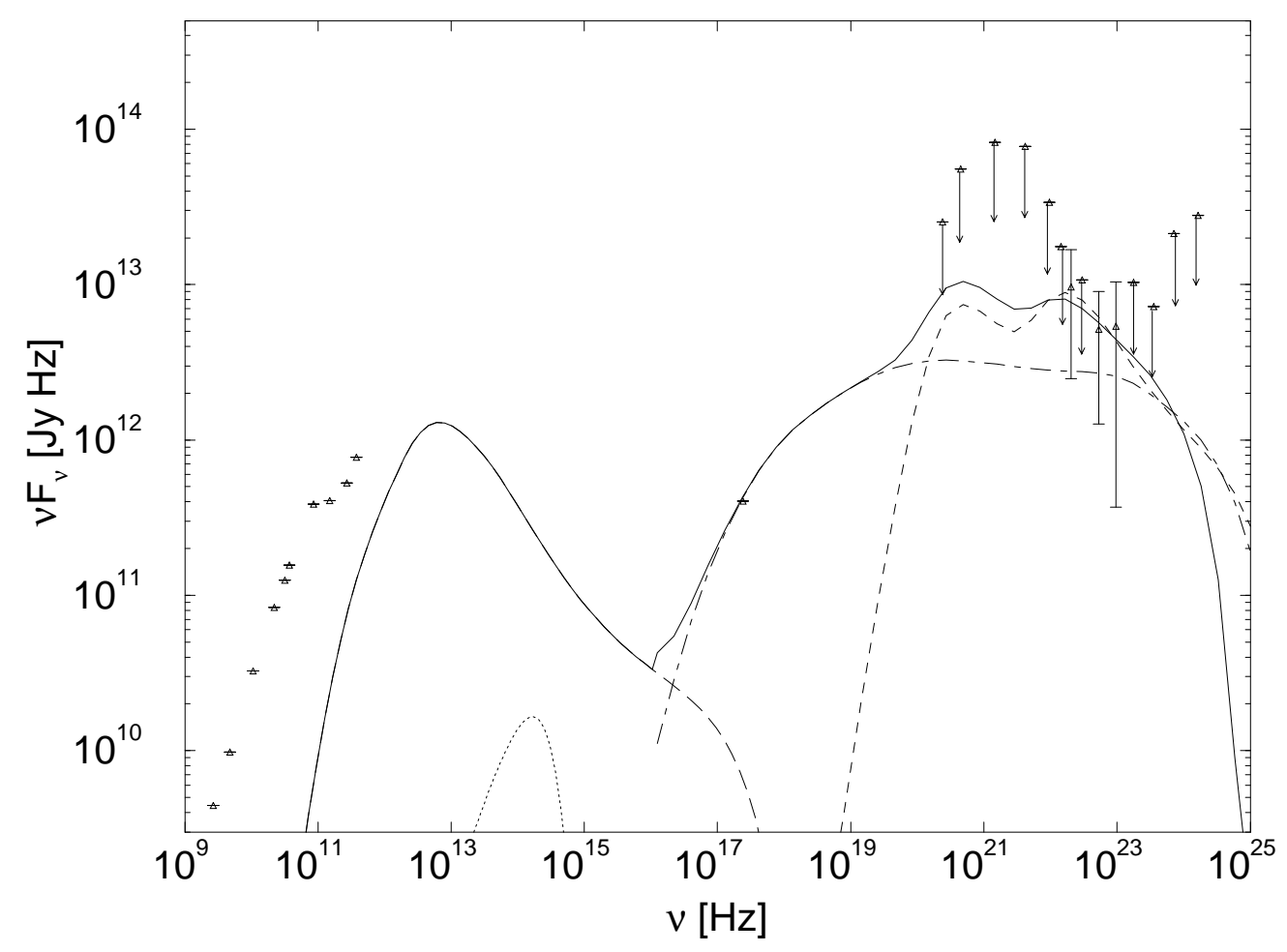

Fig. 3. - Fit to the spectral energy distribution of PKS 0528+134 in VP 39. The long-dashed line represents the synchrotron spectrum, the short-dashed line is the ERC component, the dot-dashed line is the SSC component, and the solid line represents the combined SSC + ERC model. The dotted line is the accretion disk spectrum. Parameters: $\gamma_{1}=10^{3}, \gamma_{2}=10^{5}, s=2.5, n_{e}=150$ $\mathrm{cm}^{-3}, B=2.5 \mathrm{G}, \Gamma=5$. 


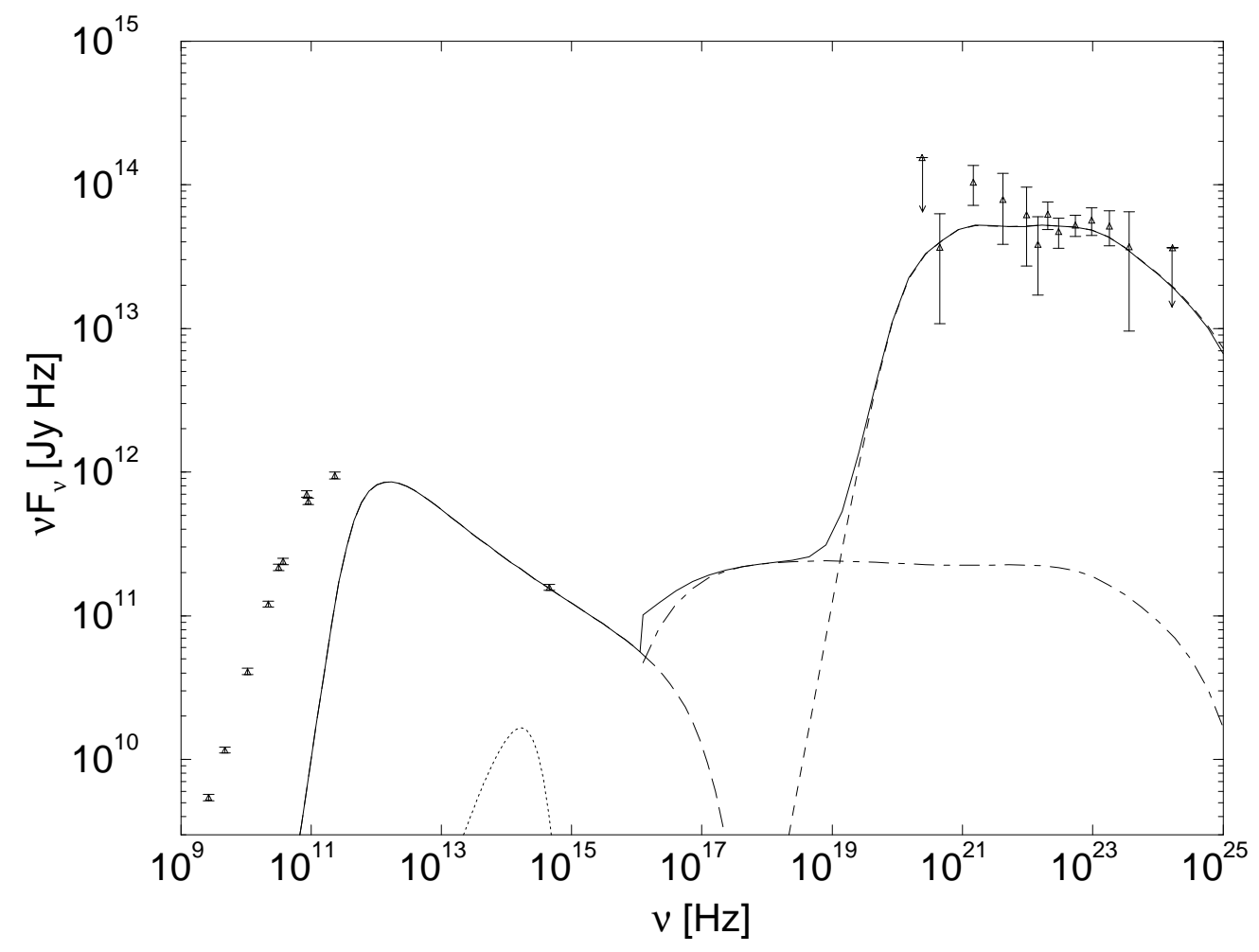

Fig. 4. - Fit to the spectral energy distribution of PKS 0528+134 in VP 213. The long-dashed line represents the synchrotron spectrum, the short-dashed line is the ERC component, the dot-dashed line is the SSC component, and the solid line represents the combined SSC + ERC model. The dotted line is the accretion disk spectrum. Parameters: $\gamma_{1}=400, \gamma_{2}=6 \times 10^{4}, s=2.5, n_{e}=180$ $\mathrm{cm}^{-3}, B=2.0 \mathrm{G}, \Gamma=20$. 


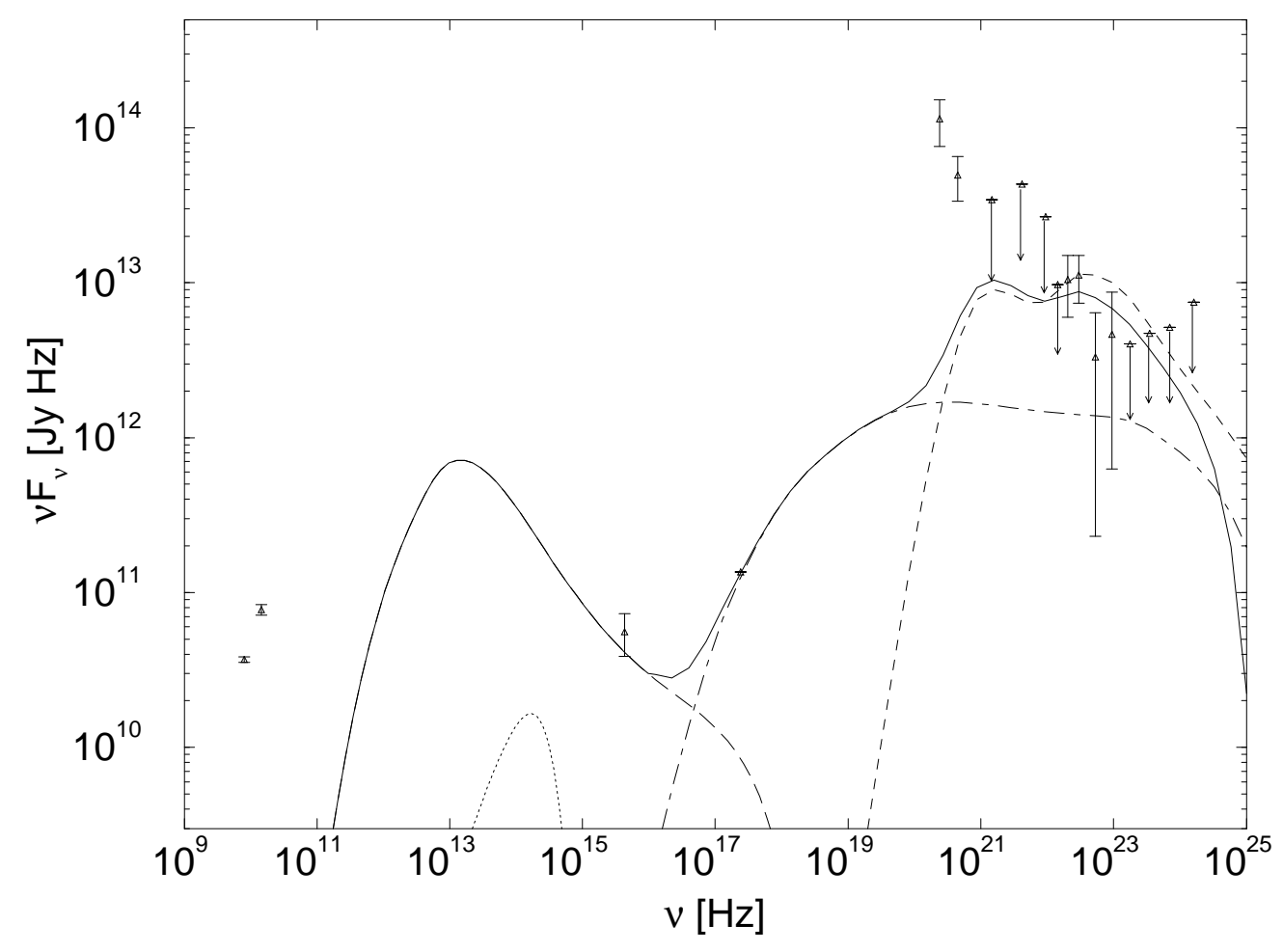

Fig. 5. - Fit to the spectral energy distribution of PKS 0528+134 in VP 337. The long-dashed line represents the synchrotron spectrum, the short-dashed line is the ERC component, the dot-dashed line is the SSC component, and the solid line represents the combined SSC + ERC model. The dotted line is the accretion disk spectrum. Parameters: $\gamma_{1}=800, \gamma_{2}=10^{5}, s=2.5, n_{e}=150$ $\mathrm{cm}^{-3}, B=3.0 \mathrm{G}, \Gamma=10$. 


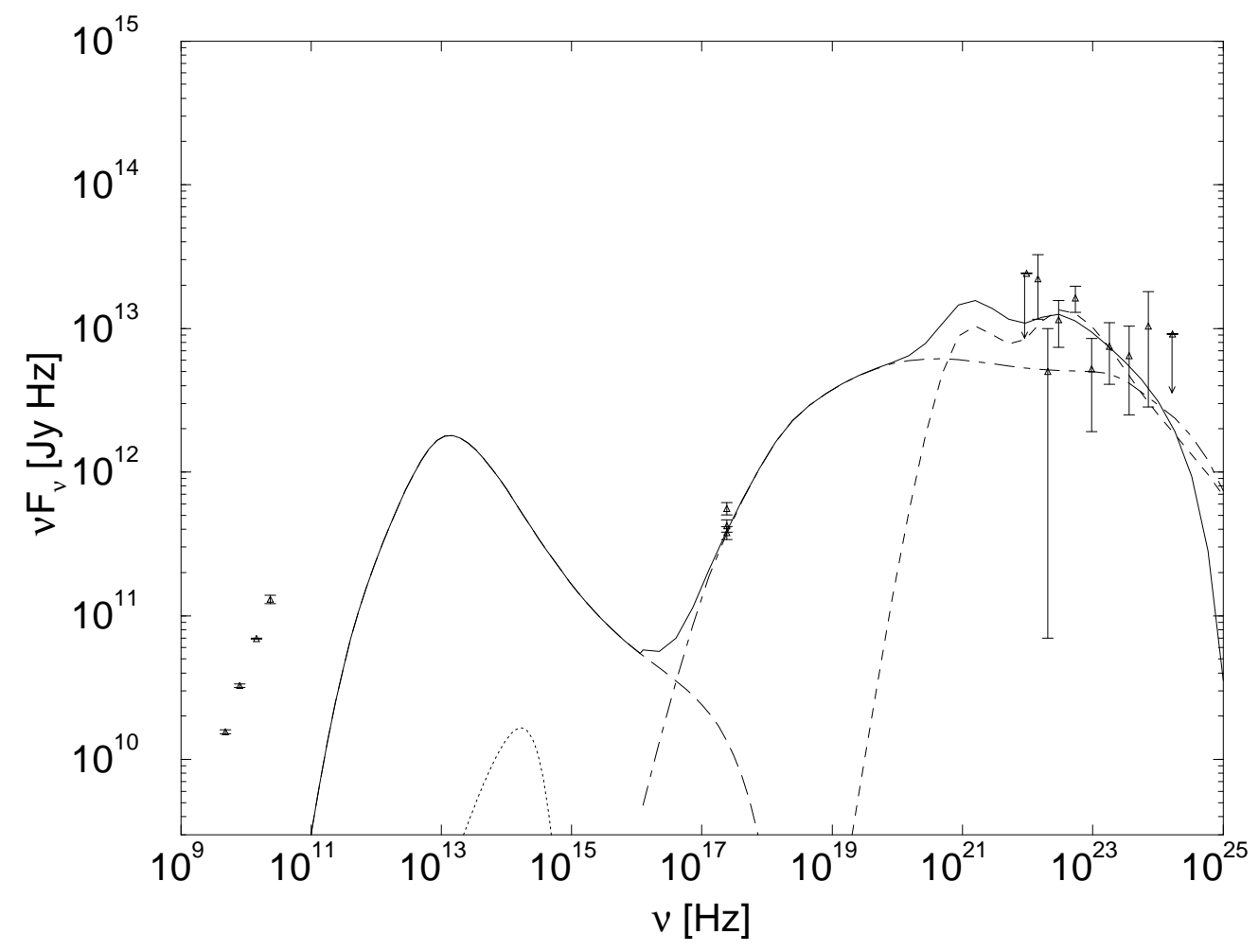

Fig. 6. - Fit to the spectral energy distribution of PKS 0528+134 in VP 413. The long-dashed line represents the synchrotron spectrum, the short-dashed line is the ERC component, the dot-dashed line is the SSC component, and the solid line represents the combined SSC + ERC model. The dotted line is the accretion disk spectrum. Parameters: $\gamma_{1}=1000, \gamma_{2}=10^{5}, s=2.5, n_{e}=180$ $\mathrm{cm}^{-3}, B=2.0 \mathrm{G}, \Gamma=10$. 


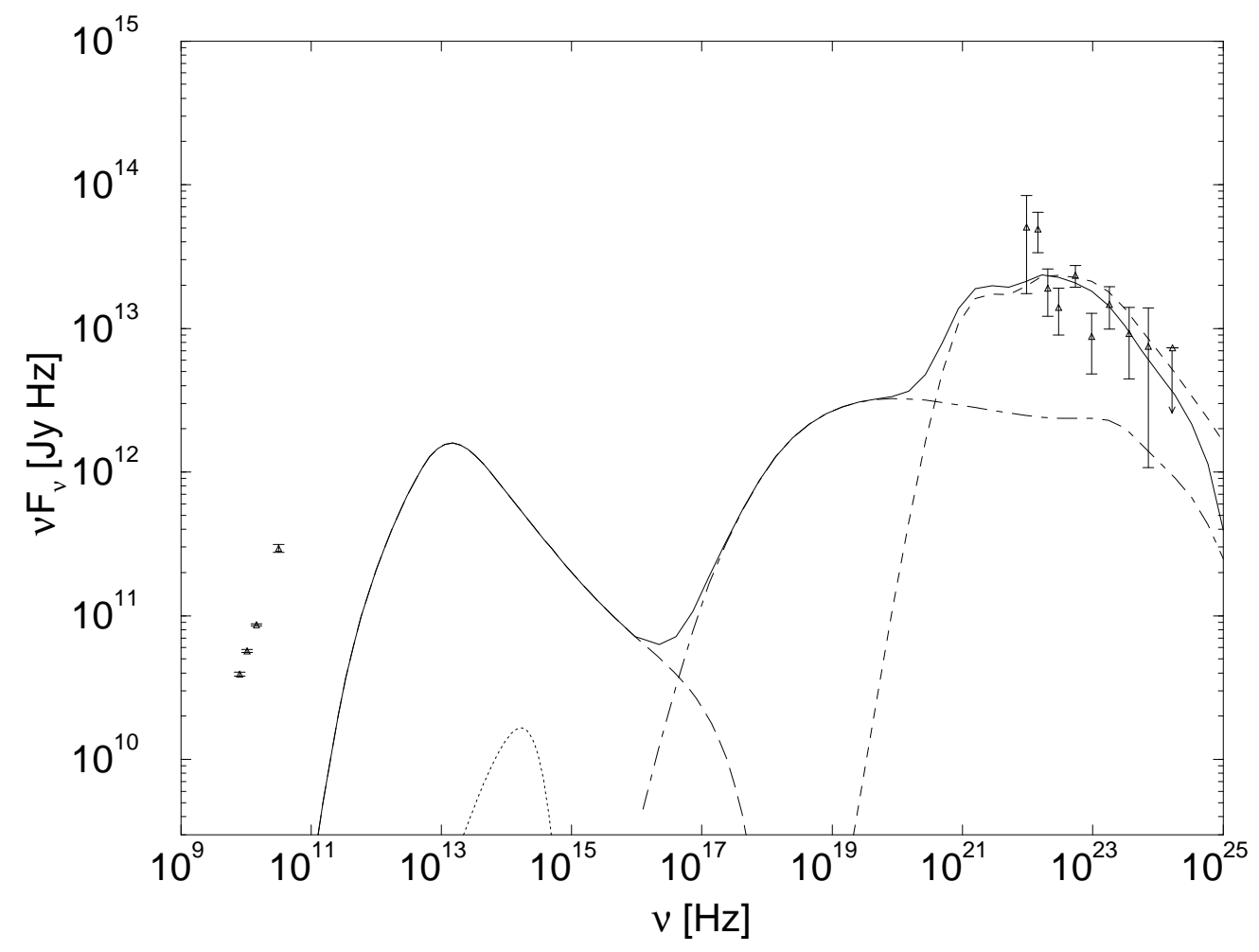

Fig. 7.- Fit to the spectral energy distribution of PKS 0528+134 in VP 420. The long-dashed line represents the synchrotron spectrum, the short-dashed line is the ERC component, the dot-dashed line is the SSC component, and the solid line represents the combined SSC + ERC model. The dotted line is the accretion disk spectrum. Parameters: $\gamma_{1}=500, \gamma_{2}=7 \times 10^{4}, s=2.5, n_{e}=180$ $\mathrm{cm}^{-3}, B=2.0 \mathrm{G}, \Gamma=20$. 


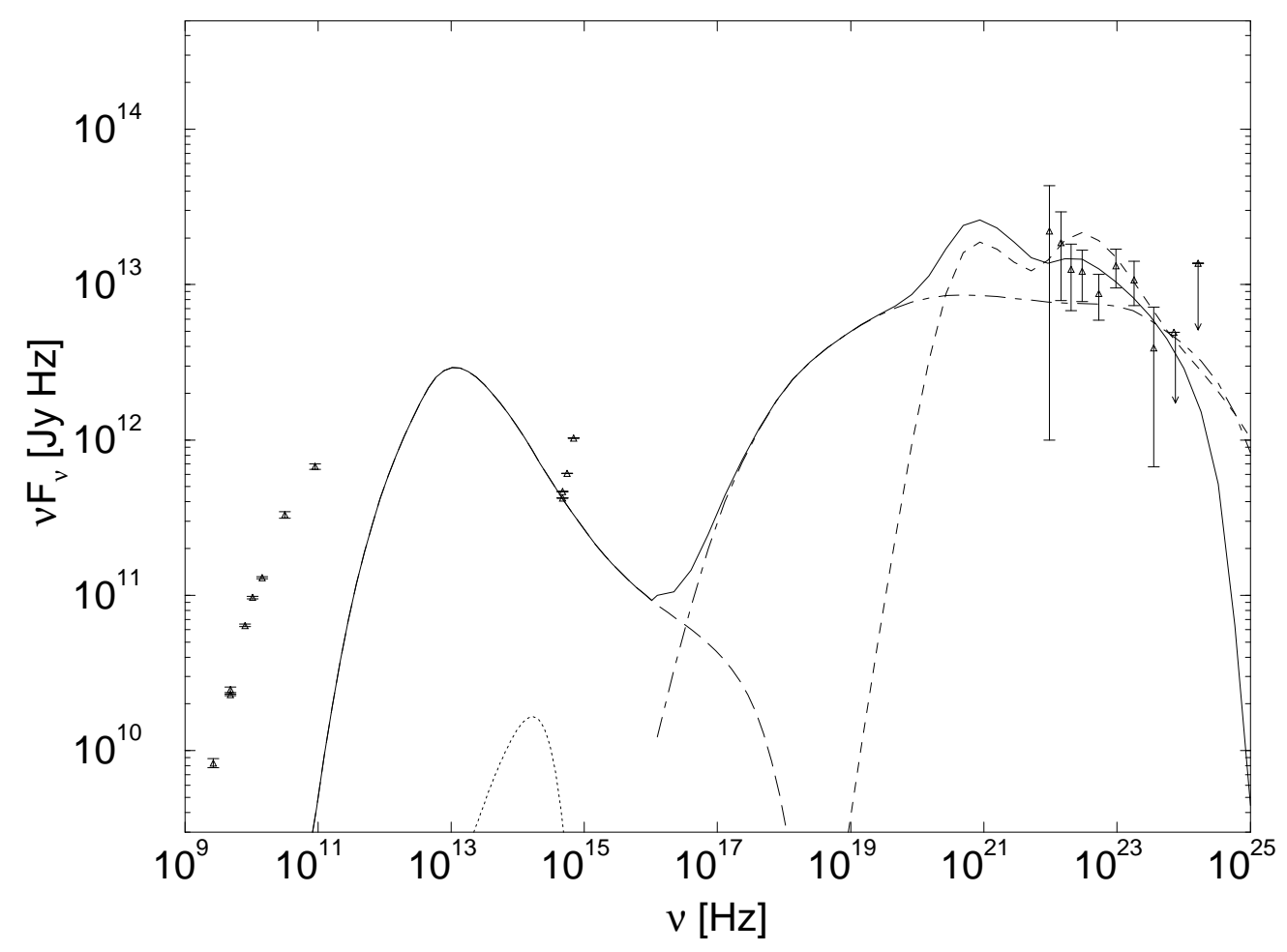

Fig. 8. - Fit to the spectral energy distribution of PKS 0528+134 in VP 502. The long-dashed line represents the synchrotron spectrum, the short-dashed line is the ERC component, the dot-dashed line is the SSC component, and the solid line represents the combined SSC + ERC model. The dotted line is the accretion disk spectrum. Parameters: $\gamma_{1}=1000, \gamma_{2}=10^{5}, s=2.5, n_{e}=180$ $\mathrm{cm}^{-3}, B=3.2 \mathrm{G}, \Gamma=7$. 


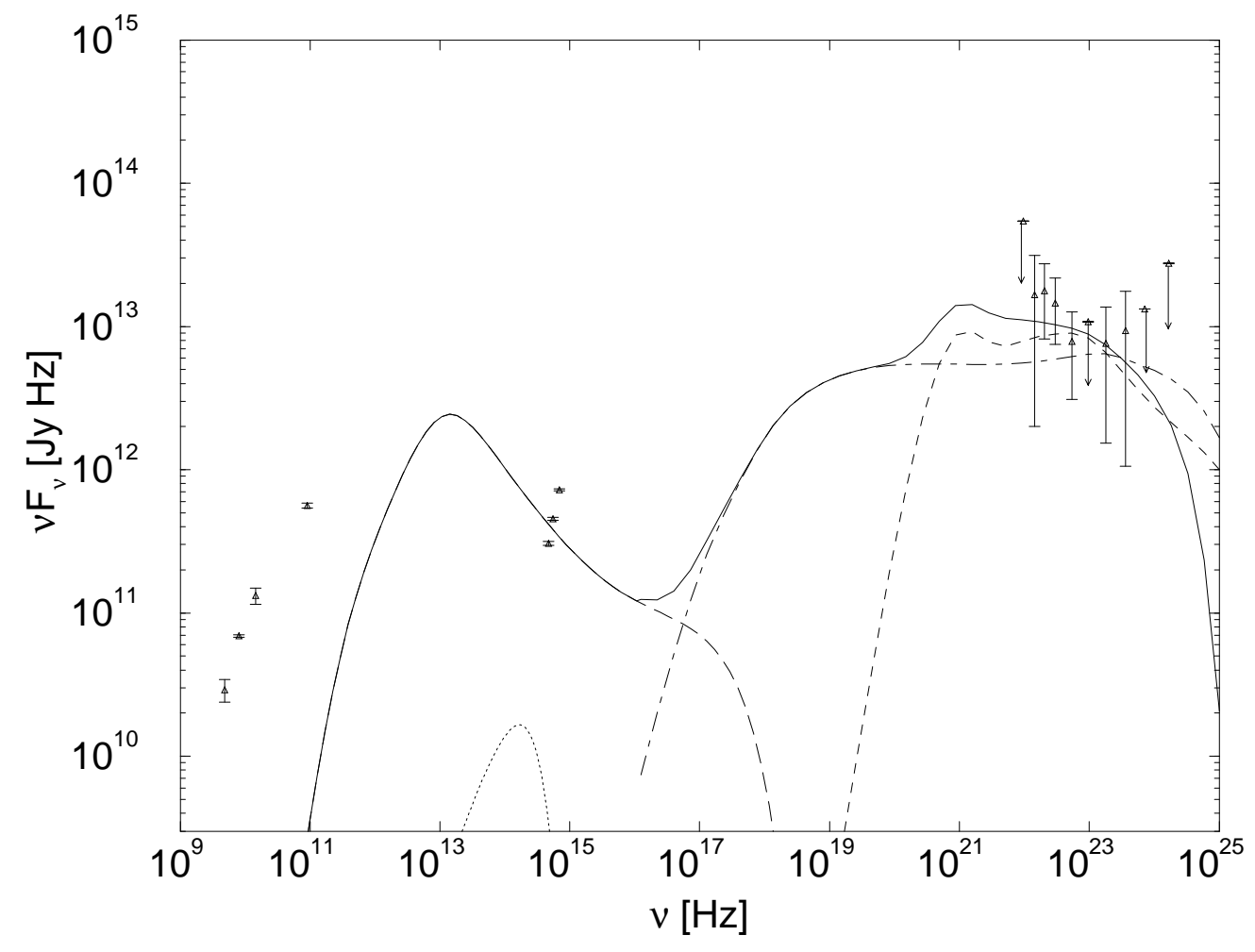

Fig. 9. - Fit to the spectral energy distribution of PKS 0528+134 in VP 528. The long-dashed line represents the synchrotron spectrum, the short-dashed line is the ERC component, the dot-dashed line is the SSC component, and the solid line represents the combined SSC + ERC model. The dotted line is the accretion disk spectrum. Parameters: $\gamma_{1}=600, \gamma_{2}=10^{5}, s=2.2, n_{e}=180$ $\mathrm{cm}^{-3}, B=2.5 \mathrm{G}, \Gamma=10$. 


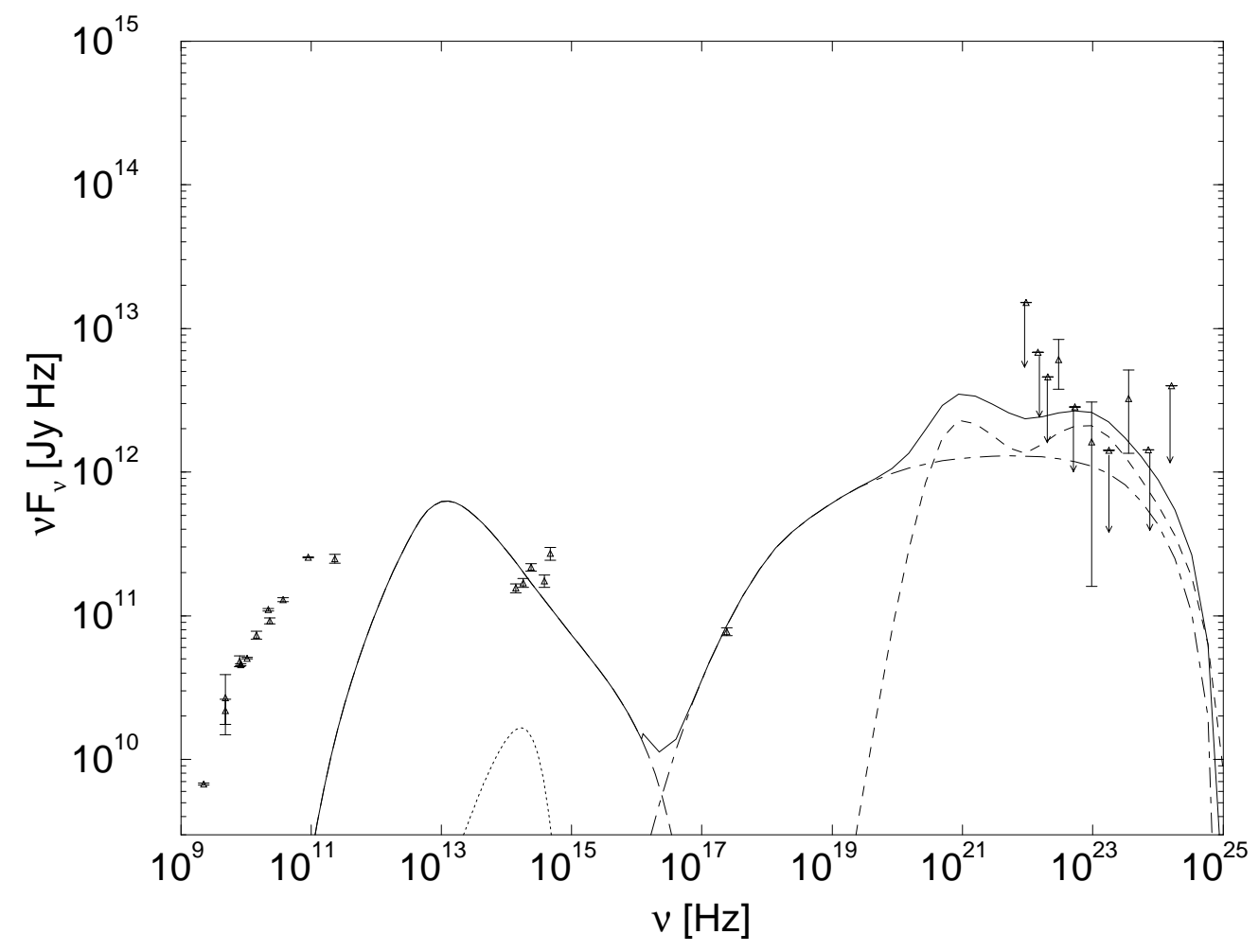

Fig. 10.- Fit to the spectral energy distribution of PKS 0528+134 in VP 616.1. The long-dashed line represents the synchrotron spectrum, the short-dashed line is the ERC component, the dotdashed line is the SSC component, and the solid line represents the combined SSC + ERC model. The dotted line is the accretion disk spectrum. Parameters: $\gamma_{1}=1000, \gamma_{2}=10^{5}, s=2.5, n_{e}=50$ $\mathrm{cm}^{-3}, B=2.5 \mathrm{G}, \Gamma=5$. 


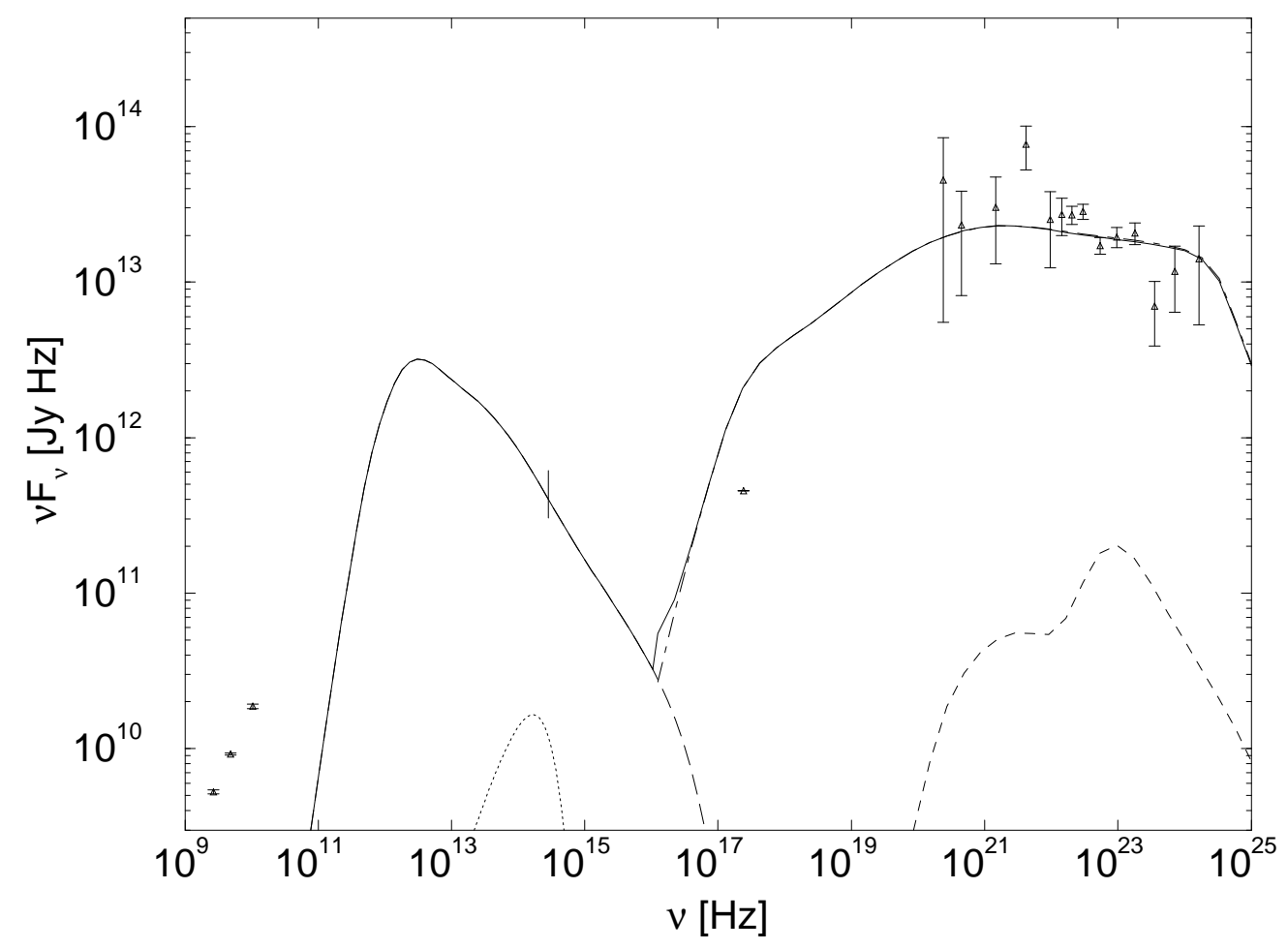

Fig. 11. - Attempt to fit to the spectral energy distribution of PKS 0528+134 in VP 0.2-0.5 with a pure SSC model. The long-dashed line represents the synchrotron spectrum, the short-dashed line is the ERC component, the dot-dashed line is the SSC component, and the solid line represents the combined SSC + ERC model. The dotted line is the accretion disk spectrum. Parameters: $\gamma_{1}=2 \times 10^{3}, \gamma_{2}=5 \times 10^{4}, s=2.5, n_{e}=200 \mathrm{~cm}^{-3}, B=0.13 \mathrm{G}, \Gamma=95, \theta_{\text {obs }}=0.6^{\circ}$, and $z_{i}=10$ pc. 


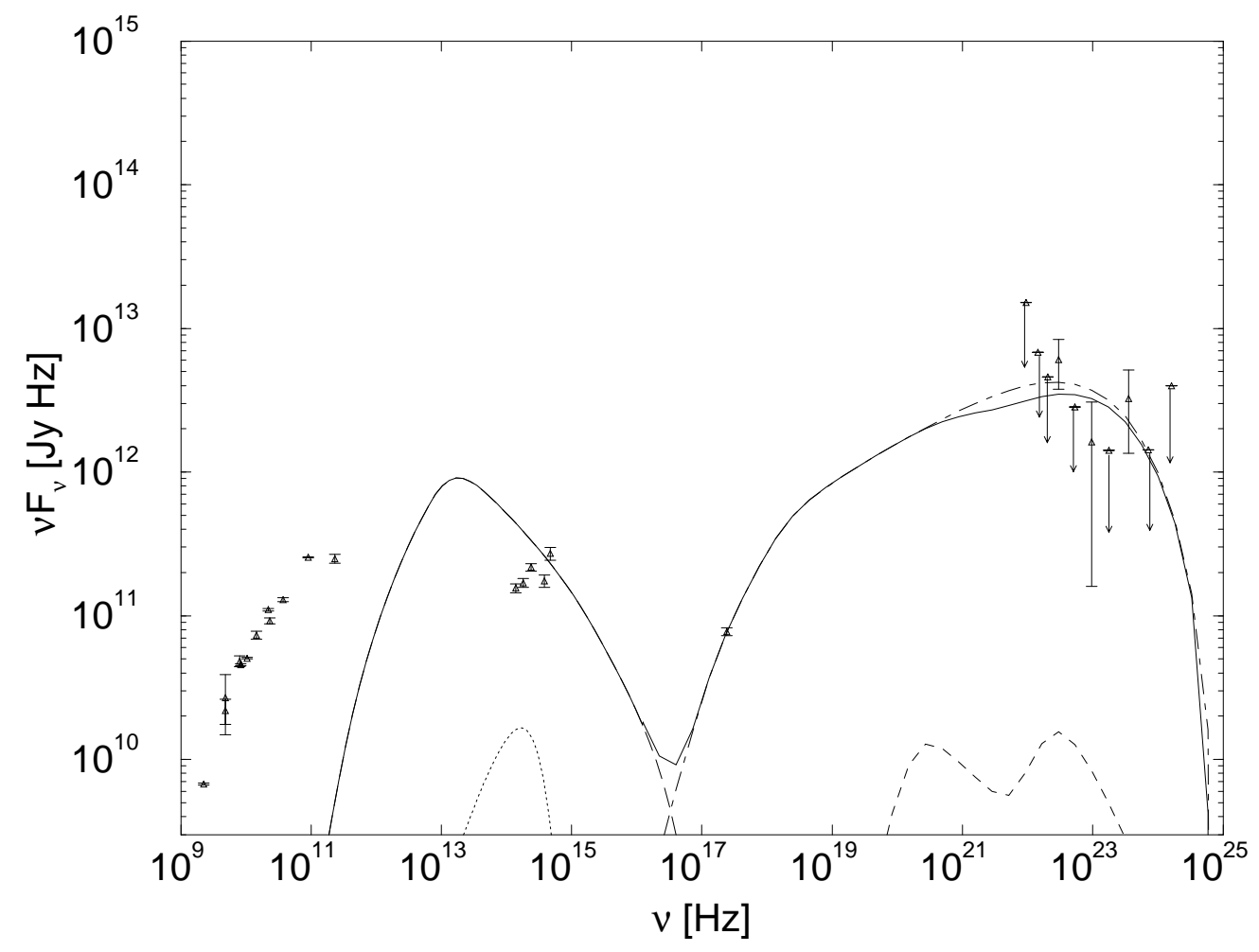

Fig. 12.- Attempt to fit to the spectral energy distribution of PKS $0528+134$ in VP 616.1 with a pure SSC model. The long-dashed line represents the synchrotron spectrum, the short-dashed line is the ERC component, the dot-dashed line is the SSC component, and the solid line represents the combined SSC + ERC model. The dotted line is the accretion disk spectrum. Parameters: $\gamma_{1}=3 \times 10^{3}, \gamma_{2}=2 \times 10^{4}, s=2.8, n_{e}=100 \mathrm{~cm}^{-3}, B=3.3 \mathrm{G}, \Gamma=10$, and $z_{i}=1 \mathrm{pc}$. 